\title{
Discrete Painlevé Equations and their Appearance in Quantum Gravity
}

\author{
A. S. Fokas ${ }^{1}$, A. R. Its ${ }^{1 \star}$ and A. V. Kitaev ${ }^{2}$ \\ 1 Department of Mathematics and Computer Science and Institute for Nonlinear Studies, \\ Clarkson University, Potsdam, New York 13699-5815, USA \\ ${ }^{2}$ Department of Mathematics, Institute of Aircraft Instrument Engineering, Gertzen 67, \\ Leningrad, 190000, USSR
}

Received March 6, 1991

\begin{abstract}
We discuss an algorithmic approach for both deriving discrete analogues of Painlevé equations as well as using such equations to characterize "similarity" reductions of spatially discrete integrable evolution equations. As a concrete example we show that a discrete analogue of Painlevé I can be used to characterize "similarity" solutions of the Kac-Moerbeke equation. It turns out that these similarity solutions also satisfy a special case of Painlevé IV equation. In addition we discuss a methodology for obtaining the relevant continuous limits not only at the level of equations but also at the level of solutions. As an example we use the WKB method in the presence of two turning points of the third order to parametrize (at the continuous limit) the solution of Painleve $\mathbf{I}$ in terms of the solution of discrete Painleve I. Finally we show that these results are useful for investigating the partition function of the matrix model in $2 \mathrm{D}$ quantum gravity associated with the measure $\exp \left[-t_{1} z^{2}-t_{2} z^{4}-t_{3} z^{6}\right]$.
\end{abstract}

\section{Introduction}

Painleve and Gambier [1], at the turn of the century, classified second order ODE's linear in the second derivative, whose solutions are free from movable branch points and essential singularities [2]. They found that, within a Möbius transformation, there exist fifty such equations. These equations can either be integrated in terms of known functions or can be reduced to one of six distinguished equations, called the six Painleve transcendents. It is quite remarkable that although the Painleve equations were introduced from purely mathematical considerations, they began, since the early 70's, appearing in several physical applications [3-4]. The appearance of these equations in physics (in particular in connection with the Ising model) and the realization that they are also closely related to integrable PDE's

\footnotetext{
* On leave of absence from Leningrad University, Leningrad, USSR
} 
[5] (like the Korteweg-deVries equation) generated a renewed interest in them. This led to the discovery $[6,7]$ of a new method, the so-called inverse monodromic (or isomonodromic) method, for studying equations of the Painlevé type. We recall that integrable PDE's can be solved using the inverse scattering (or isospectral) method. The isomonodromic method can be thought of as an extension of the isospectral method for solving certain ODE's. Both these methods reduce the solution of nonlinear equations to the solution of linear Riemann-Hilbert (RH) problems. A rigorous methodology for studying the $\mathrm{RH}$ problems associated with the isomonodromy method is given in [8].

In this paper, motivated from the recent appearance of Painleve equations in 2D quantum gravity [9-16], we study discrete versions of Painlevé equations. In particular:

(a) We shall show that there exist discrete equations which are integrable (in the sense that they admit a Lax pair representation) and furthermore they reduce to Painlevé equations in an appropriate continuous limit. Such equations, which we call discrete Painlevé equations, can also be studied via the isomonodromy approach. (b) It is well known that solutions of integrable PDE's in one spatial and one temporal dimensions invariant under the action of Lie groups, satisfy ODE's of Painlevé type. Such solutions are usually referred to as similarity solutions and the corresponding ODE's as similarity reductions. (The description invariant is more accurate since not all invariant solutions are self similar; however we shall use the terms similarity and invariant interchangeably.) Similarity solutions are physically important; in particular they play a fundamental role in the long time behavior of the associated PDE's. Since integrable PDE's have integrable discrete analogues it is natural to ask the following question: Is it possible to characterize "similarity" solutions for integrable spatially discrete equations? We use " " since the group theoretic approach breaks down for discrete equations. We shall show that discrete Painlevé equations can be used to answer this question affirmatively. Furthermore, similarity solutions of spatially discrete equations satisfy continuous equations of Painlevé type.

As an example of (a) above we note that the equation

$$
n+c_{3}=\frac{c_{1}}{2} w_{n}+\frac{c_{2}}{2} w_{n}\left(w_{n+1}+w_{n}+w_{n-1}\right),
$$

where $n \in \mathbb{Z}$ and $c_{1}, c_{2}, c_{3}$ are arbitrary constants, is the compatibility condition of the Lax pair

$$
\begin{gathered}
z Q_{n}=\frac{1}{2} w_{n+1}^{1 / 2} Q_{n+1}+\frac{1}{2} w_{n}^{1 / 2} Q_{n-1}, \\
Q_{n_{z}}=\left[c_{1} w_{n}^{1 / 2}+c_{2} w_{n}^{1 / 2}\left(w_{n-1}+w_{n}+w_{n+1}\right)\right] Q_{n-1}+c_{2}\left(w_{n} w_{n-1} w_{n-2}\right)^{1 / 2} Q_{n-3},
\end{gathered}
$$

where $z \in \mathbb{C}$. Furthermore, since the continuous limit $([10,11])$,

$$
w_{n}=-\frac{c_{1}}{6 c_{2}}\left(1-2 \varepsilon^{2} u(\xi)\right), \quad n+c_{3}=-\frac{1}{36} \frac{c_{1}^{2}}{c_{2}}\left(\frac{1}{2}+\frac{\varepsilon^{4}}{3} \xi\right), \quad \varepsilon \rightarrow 0,
$$

maps Eq. (1.1) to the Painlevé I equation

$$
\frac{d^{2} u}{d \xi^{2}}=6 u^{2}+\xi
$$

We call Eq. (1.1) discrete PI. 
As an example of (b) above we consider the Kac-Moerbeke (KM) [17] equation, a discrete analogue of the Korteweg-deVries $(\mathrm{KdV})$ equation. Let us first review the continuous case: The Lie group of transformations $x^{\prime}=x+12 \beta t$, $t^{\prime}=t+\beta, q^{\prime}=q-\beta$ (which is a combination of time translation and the Galilean group) leaves the $\mathrm{KdV}$ equation invariant; this group implies the transformation $u(\xi)=q(x, t)+t, \xi=x-6 t^{2}$, which in turn maps $\mathrm{KdV}$ to PI. It is important to notice that there exists an alternative characterization of these invariant solutions: These solutions satisfy simultaneously the $\mathrm{KdV}$ and the invariant condition $q_{t}+12 t q_{x}+1=0$; using $\mathrm{KdV}$ to eliminate $q_{t}$ we find that $q$ solves simultaneously the $\mathrm{KdV}$

$$
q_{t}+q_{x x x}-12 q q_{x}=0
$$

and the equation

$$
q_{x x x}+12(q+t) q_{x}-1=0 .
$$

This $q$ can also be found from

$$
q(x, t)=u(\xi)-t, \quad \xi=x-6 t^{2}
$$

where $u(\xi)$ solves the PI equation (1.4). We note that (1.6), when viewed as an ODE in $x$ ( $t$ considered as a fixed parameter), is also Painlevé I. We now consider the discrete versions of Eqs. (1.5) and (1.6). "Similarity" solutions of KM equations are characterized by the simultaneous solution of the KM

$$
w_{n_{t}}+w_{n}\left(w_{n+1}-w_{n-1}\right)=0
$$

and of the discrete PI equation

$$
n+\gamma=2 t w_{n}+w_{n}\left(w_{n}+w_{n+1}+w_{n-1}\right) \text {. }
$$

This $w_{n}$ can also be found by solving the PIV equation

$$
w_{n t t}=\frac{w_{n_{t}}^{2}}{2 w_{n}}+\frac{3}{2} w_{n}^{3}+4 t w_{n}^{2}+2 w_{n}\left(t^{2}+\frac{(n+\gamma)^{2}}{2}\right)-\frac{(n+\gamma)^{2}}{2 w_{n}} .
$$

Equation (1.9) is a special case of (1.1) $\left(c_{1}=4 t, c_{2}=2, c_{3}=\gamma\right)$. This example illustrates the remarkable fact that similarity reductions of both continuous (like $\mathrm{KdV}$ ) and discrete (like KM) integrable equations yield Painlevé equations.

Appropriate continuous limits of Eqs. (1.8) and (1.9) yield Eqs. (1.5) and (1.6) respectively. This implies that there must exist an appropriate limit from Eq. (1.10) to Eq. (1.4). Indeed the double limit

$$
n \rightarrow \infty, \quad t \rightarrow \infty, \quad \frac{t^{2}}{n}=O(1)
$$

maps PIV (Eq. (1.10)) to PI (Eq. (1.4)). Clearly, these kind of limits can be discussed independently of the connection with $\mathrm{KM}$ and $\mathrm{KdV}$. Actually such limits at the level of equations are given in [2]. In Sect. 4 we shall discuss the limit (1.11) at the level of solutions. Similar types of limits have also been discussed in [20].

Recalling the physical importance of similarity solutions for continuous equations, we expect that similarity solutions of discrete equations will also be significant in applications. Indeed, Eq. (1.9) with $\gamma=0$ has recently appeared in the theory of $2 \mathrm{D}$ quantum gravity and as it was mentioned earlier, provided the motivation for 
the present study. In the work of $[10,11]$ the question of computing a certain partition function reduces to the question of evaluating $h_{n}$, where $h_{n}$ is the volume element associated with the orthogonal polynomials $P_{n}(z)$, defined by the orthogonality relations

$$
\delta_{n m} h_{n}(t)=\int_{-\infty}^{\infty} P_{n}(t, z) P_{m}(t, z) e^{-4\left(t z^{2}+z^{4}\right)} d z, \quad P_{n}(t, z)=z^{n}+\cdots .
$$

Using Eq. (1.12), and defining $Q_{n}, w_{n}$ by

$$
Q_{n} \doteqdot \frac{P_{n}}{h_{n}^{1 / 2}}, \quad w_{n} \doteqdot 4 \frac{h_{n}}{h_{n-1}},
$$

it is easy to show (see Sect. 5 where we follow $[10,11]$ ) that $w_{n}$ satisfies Eqs. (1.8) and (1.9) with $\gamma=0$, while $Q_{n}$ satisfy Eqs. (1.2) and (1.3) with $c_{1}=4 t, c_{2}=2$. Also

$$
w_{n}=0 \text { for } n=0,-1,-2, \ldots, \text { and } w_{1}=4 \frac{\int_{-\infty}^{\infty} z^{2} e^{-4\left(t z^{2}+z^{4}\right)} d z}{\int_{-\infty}^{\infty} e^{-4\left(t z^{2}+z^{4}\right)} d z} \text {. }
$$

Using this fact we show in Sect. 3 that it is possible to express $w_{n}$ (and hence $h_{n}$ ) in terms of certain derivatives of parabolic cylinder functions. Furthermore the results on continuous limits derived in Sect. 4 are used to discuss the physical significance of these solutions.

In more details this paper is organized as follows:

In Sect. 2 we give an algorithmic approach for both deriving discrete analogues of Painlevé equations (such as Eq. (1.1)) as well as using these equations to characterize "similarity" solutions of spatially discrete integrable evolution equations.

In Sect. 3 we use the isomonodromy approach to express the solution $w_{n}(t)$ of the PIV equation (1.10) in terms of appropriate monodromy data $\{S\}$. We recall that $w_{n}(t)$ is the "similarity" solution of the KM equation (1.8), characterized by the fact that it satisfies simultaneously Eq. (1.8) and the discrete PI equation (1.9). For a special choice of the monodromy data, the so-called triangular case, it is shown that $w_{n}(t)$ can be expressed in terms of parabolic cylinder functions.

In Sect. 4 we first show that the transformations

$$
w_{n}=-\frac{t}{3}\left(1-2 h^{2} u(\xi)\right), \quad \frac{n}{t^{2}}=-\frac{1}{3}\left(1+\frac{2}{3} h^{4} \xi\right), \quad t^{2}=-\frac{9}{2} h^{-5}, \quad h \rightarrow 0,
$$

map both the discrete PI equation (1.9) with $\gamma=0$, and the KM equation (1.8), to the PI equation (1.4). Furthermore, the transformations

$$
Q_{n \pm 1} e^{-2 t z^{2}-2 z^{4}}=\psi(\xi \pm h), \quad z=\left(-\frac{t}{3}\right)^{1 / 2}\left(1+\frac{h^{2} k^{2}}{2}\right), \quad h \rightarrow 0,
$$

map the Lax pair of the discrete PI (Eqs. (1.2) and (1.3)) to the Lax pair of PI $(\psi(\xi, k)$ denotes the eigenfunction associated with PI).

In Sect. 4.1 using the methodology of [27] we investigate the above limits at the level of solutions. We consider only the case of $t$ imaginary

$$
t=i \tau, \quad \tau>0,
$$

and we scale $z$ by $z=\sqrt{\tau \zeta}$. Let $\Phi_{n}(\tau, \zeta)$ and $Y(\xi, k)$ denote the matrix eigenfunctions 
associated with $w_{n}(\tau)$ and $u(\xi)$ respectively. For the complete description of $w_{n}$ and $u$ one needs 8 and 10 such matrices respectively; since these matrices will be related asymptotically, we denote them with superscripts $j$ and $f(j)$ respectively. Let $\{S\}$ and $\{G\}$ denote the monodromy data associated with $\Phi_{n}$ and $Y$ respectively. As $\tau \rightarrow \infty, \Phi_{n}^{(j)}$ can be found by using the WKB method, which in this particular case involves two turning points $\zeta_{+}=\frac{e^{-i \pi / 4}}{\sqrt{3}}$ and $\zeta_{-}=-\frac{e^{-i \pi / 4}}{\sqrt{3}}$. The matrix solutions $\Phi_{n}^{(j)}, j=1,2,7,8$ can be expressed in terms of WKB solutions $\Phi_{n}$ near $\zeta_{+}$; similarly the matrices for $j=3,4,5,6$ can be expressed in terms of WKB solutions near $\zeta_{-}$(see Fig. 4.1). Near the turning point $\zeta_{+}$, the WKB solutions $\Phi_{n}$, $T P(j)$

$j=1,2,7,8$ break down and are replaced by $\Phi_{n}, j=1,2,7,8$. It is shown in Sect. 4.1 $T P(j)$

that $\Phi_{n}$ can be related to $Y^{f(j)}$, with $f(1)=1, f(2)=2, f(7)=-1, f(8)=0$ (similarly $T P(j) \quad$ WKB

the $\Phi_{n}, j=3,4,5,6$ are also related to appropriate $Y$ 's). Since $\Phi_{n}$ is related to $T P$

$\Phi_{n}$ the above analysis provides a connection between the matrices $\Phi_{n}$ 's and the matrix $Y$ 's. Using this connection we find that the associated monodromy data satisfy

$$
S_{j}=e^{\alpha_{0} \sigma_{3}} G_{f(j)} \sigma_{3} e^{-\alpha_{0} \sigma_{3}}, \quad j=1,7,8,
$$

where $\alpha_{0}$ is a certain constant.

Let $s$ and $g$ denote the entries of the monodromy matrices $S$ and $G$ respectively. In the special triangular case considered in Sect. 4.1 our analysis summarized above yields

$$
g_{5}=0, \quad g_{2}=g_{3}=i, \quad g_{1}=\frac{i}{1+p}, \quad g_{4}=\frac{i p}{1+p}, \quad p \doteqdot \frac{s_{3}}{s_{1}}, \quad \alpha_{0}=\frac{1}{2} \ln \frac{i s_{1}^{2}}{s_{3}-s_{1}} .
$$

In Sect. 5 we show that the $w_{n}(t)$ corresponding to the triangular case studied in Sect. 3.1, is associated with the $2 \mathrm{D}$ quantum gravity corresponding to the measure $e^{-4\left(t z^{2}+z^{4}\right)}$ and discuss the physical meaning of the results of Sect. 4.1 concerning the calculation of the parameters of the PI-function $u(\xi)$.

Some of the results of Sect. 5 we were announced in [29] and were also obtained in $[14]$

\section{Discrete Analogues of Painlevé Equations and Similarity Reductions of Spatially Discrete Integrable Equations}

As it was mentioned in the introduction, there exist integrable discrete analogues of the Painlevé equations (see for example Eq. (1.1)). Such equations can be used to characterize similarity reductions of spatially discrete integrable equations. Since, in this paper we are mainly concerned with this particular application of discrete Painlevé equations, we concentrate on how to find a discrete Painlevé equation associated with a given spatially discrete integrable equation. For this purpose we propose the following algorithmic approach.

Suppose we are given a spatially discrete integrable evolution equation in one spatial dimension, and its associated Lax pair. To find a "similarity" reduction of 
this equation we first find an appropriate discrete Painleve equation. This is achieved by looking for an equation of the form $Q_{n_{\mathrm{z}}}=F\left(Q_{n}, Q_{n+1}, \ldots, Q_{n-1}, \ldots\right)$ which is compatible with the $t$-independent part of the given Lax pair. The compatibility condition of the $t$-independent part of the Lax pair and of $Q_{n_{z}}=F$ defines a discrete Painlevé equation. This equation will depend in general on a number of constant in $n$ parameters. We then choose these parameters so that $Q_{n_{z}}=F$ is also compatible with the $t$-part of the given Lax pair. The discrete Painleve equation with this choice of parameters is compatible with the original spatially discrete equation. Hence, the simultaneous solution of these equations characterizes a "similarity" reduction of the given spatially discrete evolution equation. We emphasize that the above procedure is completely algorithmic. Also in the examples we have studied it is possible to manipulate the above two equations to obtain a single ODE.

Example. Consider the KM equation (1.8); its Lax pair is given by Eq. (1.2) and by

$$
Q_{n_{t}}=\frac{1}{2}\left(w_{n}+w_{n+1}\right) Q_{n}+w_{n}^{1 / 2} w_{n-1}^{1 / 2} Q_{n-2} .
$$

In order to find a discrete Painleve equation we consider the compatibility of Eq. (1.2) and of $Q_{n_{z}}=F$. Let for example $F=F\left(Q_{n-1}, Q_{n-3}\right)$ (other choices of $F$ will give us "higher" Painlevé equations). That is, we consider

$$
Q_{n_{z}}=A_{n} Q_{n-1}+B_{n} Q_{n-3} \text {. }
$$

Demanding that Eqs. (1.2) and (2.2) are compatible we obtain three equations; these equations are obtained by equating the coefficients of $Q_{n-4}, Q_{n-2}$, and $Q_{n}$ to zero. The coefficient of $Q_{n-4}$ yields

$$
\frac{B_{n}}{w_{n}^{1 / 2}}=\frac{B_{n-1}}{w_{n-3}^{1 / 2}}
$$

This equation can be written as

$$
\frac{B_{n}}{\left(w_{n} w_{n-1} w_{n-2}\right)^{1 / 2}}=\frac{B_{n-1}}{\left(w_{n-1} w_{n-2} w_{n-3}\right)^{1 / 2}},
$$

and hence it implies $B_{n}=c_{2}\left(w_{n} w_{n-1} w_{n-2}\right)^{1 / 2}$. Using this expression for $B_{n}$, the coefficient of $Q_{n-2}$ yields

$$
\frac{A_{n}}{\left(w_{n}\right)^{1 / 2}}-c_{2} w_{n+1}=\frac{A_{n-1}}{\left(w_{n-1}\right)^{1 / 2}}-c_{2} w_{n-2} .
$$

Adding to both sides of this equation the term $-c_{2} w_{n}-c_{2} w_{n-1}$, it follows that

$$
\frac{A_{n}}{\left(w_{n}\right)^{1 / 2}}=c_{1}+c_{2}\left(w_{n-1}+w_{n}+w_{n+1}\right) \text {. }
$$

Using these expressions for $A_{n}$ and $B_{n}$, Eq. (2.2) becomes Eq. (1.3); also the coefficient of $Q_{n}$ implies Eq. (1.1).

Demanding that Eqs. (2.1) and (1.3) are compatible we find $c_{1}=4 t, c_{2}=2$, and $c_{3}=\gamma$; hence Eq. (1.1) reduces to Eq. (1.9).

The similarity reduction of the KM equation, characterized by the simultaneous solution of the KM equation (1.8) and of the discrete Painleve equation (1.1) can 
be characterized further: Equations (1.1) and (1.8) can be written in the form

$$
\begin{aligned}
& 2 w_{n+1}=\frac{n}{w_{n}}-\frac{w_{n_{t}}}{w_{n}}-2 t-w_{n}, \\
& 2 w_{n-1}=\frac{n}{w_{n}}+\frac{w_{n_{t}}}{w_{n}}-2 t-w_{n} .
\end{aligned}
$$

Writing Eq. (2.3b) as,

$$
2 w_{n}=\frac{n+1}{w_{n+1}}+\frac{\left(w_{n+1}\right)_{t}}{w_{n+1}}-2 t-w_{n+1},
$$

and using Eq. (2.3a) to eliminate $w_{n+1}$ we obtain a second order ODE for $w_{n}(t)$. Since the KM equation is integrable we expect that this ODE is also integrable. Indeed, we obtain Eq. (1.10) which is a special case of Painlevé IV (PIV).

Other approaches to deriving discrete Painlevé equations are given in [18 and 19].

\section{A Method of Solution}

It was shown in Sect. 2 that a "similarity" reduction of the Kac-Moerbeke equation satisfies the PIV equation (1.10). This equation can be solved by the isomonodromy method. For the sake of completeness we indicate how Eq. (1.10) can be solved in the present context.

Letting $c_{1}=4 t, c_{2}=2, c_{3}=\gamma=0$ and

$$
\vec{\Phi}_{n}=\left(Q_{n+1}, Q_{n}\right)^{T} e^{-2 t z^{2}-2 z^{4}},
$$

and writing Eqs. (1.2), (1.3), (2.1) in matrix form we find

$$
\begin{gathered}
\Phi_{n+1}(z)=U_{n}(z) \Phi_{n}(z), \\
\Phi_{n_{t}}(z)=V_{n}(z) \Phi_{n}(z), \\
\Phi_{n_{z}}(z)=A_{n}(z) \Phi_{n}(z),
\end{gathered}
$$

where the matrices $U_{n}, V_{n}, A_{n}$ are defined by

$$
\begin{gathered}
U_{n}(z)=\left(\begin{array}{cc}
2 z w_{n+2}^{-1 / 2} & -w_{n+1}^{1 / 2} w_{n+2}^{-1 / 2} \\
1 & 0
\end{array}\right), \\
V_{n}(z)=\left(\begin{array}{cc}
-2 z^{2}+\frac{1}{2}\left(w_{n+2}-w_{n+1}\right), & 2 z w_{n+1}^{1 / 2} \\
-2 z w_{n+1}^{1 / 2}, & 2 z^{2}+\frac{1}{2}\left(w_{n+1}-w_{n}\right)
\end{array}\right),
\end{gathered}
$$

and

$$
A_{n}(z)=\left(\begin{array}{cc}
-8 z^{3}-4 t z-4 z w_{n+1}, & w_{n+1}^{1 / 2}\left(4 t+8 z^{2}+2\left(w_{n+1}+w_{n+2}\right)\right) \\
-w_{n+1}^{1 / 2}\left(4 t+8 z^{2}+2\left(w_{n}+w_{n+1}\right)\right), & 8 z^{3}+4 t z+4 z w_{n+1}
\end{array}\right)
$$

For simplicity of notation we have suppressed the explicit dependence on $t$. The transformation (3.1) was used in order to obtain a traceless matrix $A_{n}$. 
Equation (3.4) plays a fundamental role in the subsequent analysis, while Eqs. (3.2) and (3.3) play only auxiliary roles. The basic idea of the isomonodromy method consists of using Eq. (3.4) to formulate an inverse problem for $\Phi_{n}(z)$ in terms of appropriate monodromy data. This can be achieved by determining the analytic structure of solutions of Eq. (3.4) with respect to $z \in \mathbb{C}$. Since Eq. (3.4) is a linear ODE in $z$, the analytic structure of $\Phi_{n}$ depends only on $A_{n}$. Actually, Eq. (3.4) has only one singularity, namely an irregular singular point at $z=\infty$. A formal solution at $z=\infty$ has the form,

$$
\Phi_{n} \sim \Phi_{n}^{(\infty)}, \quad \Phi_{n}^{(\infty)}=\hat{\Phi}_{n}^{(\infty)} e^{-\left(2 z^{4}+2 t z^{2}-(n+1) \ln z\right) \sigma_{3}},
$$

where $\sigma_{3}=\operatorname{diag}(1,-1)$, and $\hat{\Phi}_{n}^{(\infty)}$ is a formal power series. However, the actual asymptotic behavior of $\Phi_{n}$ changes form in certain sectors of the complex $z$-plane (Stoke's phenomenon). These sectors are determined by $\operatorname{Re}\left(2 z^{4}+2 t z^{2}\right)=0$; thus for large $z$ the boundaries of the sectors which we call $\Sigma_{j}$, are asymptotic to the rays $\arg z=\frac{-\pi}{8}+\frac{2 j \pi}{8}, 0 \leqq j \leqq 7$. Let $\Omega_{j}$ be the sector containing the boundary $\Sigma_{j}$, i.e. if $z \in \Omega_{1}, \frac{-\pi}{8} \leqq \arg z<\frac{\pi}{8}$, etc. Then, if $\Phi_{n} \sim \Phi_{n}^{(\infty)}$ as $z \rightarrow \infty$ in $\Omega_{1}$, it turns out that $\Phi_{n} \sim \Phi_{n}^{(\infty)} S_{1} S_{2}, \ldots, S_{j}$, as $z \rightarrow \infty$ in $\Omega_{j+1}, 1 \leqq j \leqq 8$. The matrices $S_{j}$, $1 \leqq j \leqq 8$ are triangular and are called Stokes matrices. Alternatively, it is more convenient to introduce different solutions $\Phi_{n}^{(j)}, 1 \leqq j \leqq 9$ such that $\Phi_{n}^{(j)}$ is asymptotic to $\Phi_{n}^{(\infty)}$ in $\Omega_{j}$. Then $\Phi_{n}^{(j+1)}=\Phi_{n}^{(j)} S_{j}, 1 \leqq j \leqq 8$; also it can be shown that $\Phi_{n}^{(1)}(z)=\Phi_{n}^{(9)}\left(z e^{2 i \pi}\right) e^{2 i \pi(n+1) \sigma_{3}}=\Phi_{n}^{(9)}\left(z e^{2 i \pi}\right)$. Therefore

$$
\begin{aligned}
\Phi_{n}^{(j+1)}(z) & =\Phi_{n}^{(j)}(z) S_{j}, \quad 1 \leqq j \leqq 7, \quad \Phi_{n}^{(1)}(z)=\Phi_{n}^{(8)}\left(z e^{2 i \pi}\right) S_{8} \\
\Phi_{n}^{(j)}(z) & \sim \Phi_{n}^{(\infty)} e^{-\left(2 z^{4}+2 t z^{2}-(n+1) \ln z\right) \sigma_{3}}, \quad \text { as } \quad z \rightarrow \infty \quad \text { in } \Omega_{j} .
\end{aligned}
$$

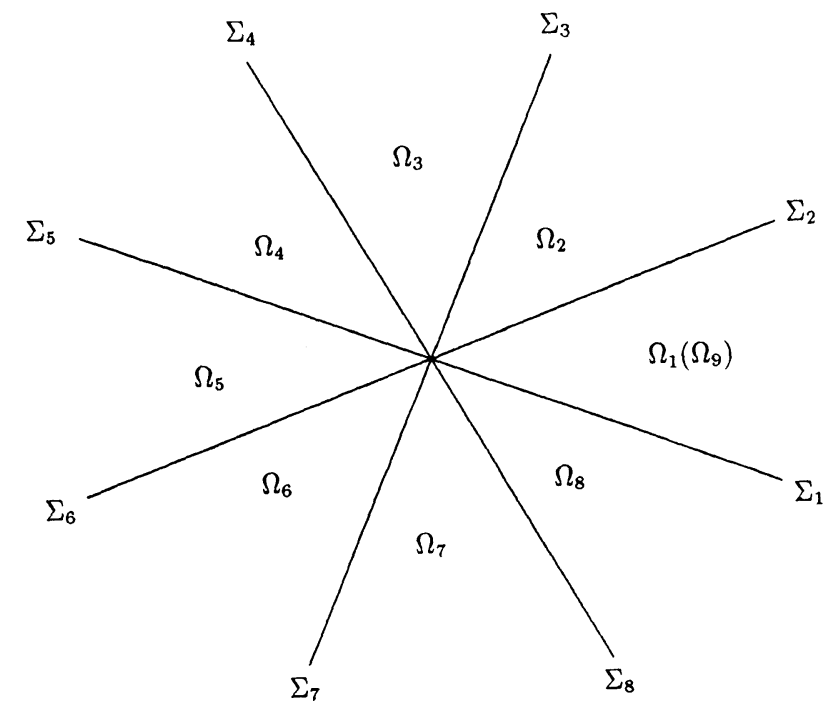

Fig. 3.1 
The Stokes matrices have the form

$$
S_{2 j+1}=\left(\begin{array}{cc}
1 & s_{2 j+1} \\
0 & 1
\end{array}\right), \quad S_{2 j}=\left(\begin{array}{cc}
1 & 0 \\
s_{2 j} & 1
\end{array}\right), \quad 0 \leqq j \leqq 4 \bmod 4
$$

Using the symmetry relationship

$$
\sigma_{3} \Phi_{n}^{(j)}(-z) \sigma_{3}=\Phi_{n}^{(j+4)}(z)(-1)^{n+1}
$$

it follows that the Stokes matrices satisfy the constraints

$$
S_{j+4}=\sigma_{3} S_{j} \sigma_{3} \text {. }
$$

Also, Eq. (3.9) implies the cyclic equality

$$
S_{1} S_{2} \cdots S_{8}=I \text {. }
$$

The constraints (3.13), (3.14) identify the set of the monodromy data as a threedimensional algebraic variety. The quantity $w_{n}$ can be reconstructed via

$$
w_{n+1}=4\left(\Phi_{n, 1}^{(\infty)}\right)_{12}\left(\Phi_{n, 1}^{(\infty)}\right)_{21},
$$

where $\Phi_{n, k}^{(\infty)}$ are the coefficients of the formal series

$$
\hat{\Phi}_{n}^{(\infty)}=\Lambda_{n}(t)\left(I+\sum_{k=1}^{\infty} \Phi_{n, k}^{(\infty)} z^{-k}\right) .
$$

Equation (3.15) implies that $w_{n}$ depends only on the orbits of the action

$$
S_{k} \mapsto \exp \left(\delta \sigma_{3}\right) S_{k} \exp \left(-\delta \sigma_{3}\right), \quad \delta \in \mathbb{C} .
$$

This action is well defined on the algebraic variety specified by Eqs. (3.13) and (3.14).

Since $A_{n}$ depends on $n$ and on $t$, it follows that the monodromy data will also depend in general on $n$ and $t$. However, it is possible to normalize $\Phi_{n}$ in such a way that, if $w_{n}$ satisfies Eqs. (1.8) and (1.9) then, the monodromy data are $n$ and $t$ independent (this is a usual situation in the isomonodromy method [7]). The correct normalization is achieved by choosing $\Lambda_{n}(t)$ so that the formal solution $\hat{\Phi}_{n}^{(\infty)}$ defined in Eq. (3.16), is also a formal solution of Eqs. (3.2) and (3.3). This is the case if

$$
\Lambda_{n}(t)=w_{n+1}^{-1 / 4} e^{H_{n} \sigma_{3}}, \quad \partial_{t} H_{n}=\frac{n+1}{4 w_{n+1}}-\frac{t}{2}+\frac{1}{4} w_{n+1}
$$

and

$$
H_{n+1}-H_{n}=\ln 2-\frac{1}{4} \ln w_{n+1} w_{n+2} .
$$

Indeed substituting $\Phi_{n}^{(\infty)}$ in Eq. (3.3), where $\hat{\Phi}_{n}^{(\infty)}$ is given by (3.16), we find

$$
\left[\sigma_{3}, \Lambda_{n} \Phi_{n, 1}^{(\infty)}\right]=w_{n+1}^{1 / 2}\left(\begin{array}{cc}
0 & 1 \\
-1 & 0
\end{array}\right) \Lambda_{n}
$$

and

$$
\partial_{t} \Lambda_{n}=-\frac{1}{2}\left[\sigma_{3}, \Lambda_{n} \Phi_{n, 2}^{(\infty)}\right]+\frac{1}{2} w_{n+1}^{1 / 2}\left(\begin{array}{cc}
0 & 1 \\
-1 & 0
\end{array}\right) \Lambda_{n} \Phi_{n, 1}^{(\infty)}+\frac{1}{8}\left(\begin{array}{cc}
w_{n+2}-w_{n+1} & 0 \\
0 & w_{n+1}-w_{n}
\end{array}\right) \Lambda_{n}
$$


These equations imply,

$$
\partial_{t} \ln \left(\Lambda_{n}\right)_{1}=\frac{1}{8}\left(w_{n+2}+w_{n+1}\right)=-\partial_{t} \ln \left(\Lambda_{n}\right)_{2} .
$$

Letting $\left(\Lambda_{n}\right)_{1}=a_{n} e^{H_{n}},\left(\Lambda_{n}\right)_{2}=a_{n} e^{-H_{n}}$, we find

$$
a_{n}=w_{n+1}^{-1 / 4}, \quad \partial_{t} H_{n}=\frac{1}{4} \partial_{t} \ln w_{n+1}+\frac{1}{8}\left(w_{n+2}+w_{n+1}\right) .
$$

Using Eqs. (1.8) and (1.9) to simplify the equation for $\partial_{t} H_{n}$ we find Eq. (3.18b). Similarly, substituting $\Phi_{n}^{(\infty)}$ in Eq. (3.2) we find Eq. (3.19).

Equations (2.3) imply that Eqs. (3.18) and (3.19) are consistent; they can be rewritten in the form

$$
H_{n}=n \ln 2-\frac{1}{4} \ln w_{n+1} w_{n}^{2} \cdots w_{2}^{2} w_{1}+H_{0}(t) ; \quad \partial_{t} H_{0}=\frac{1}{4}\left(w_{1}+\frac{1}{w_{1}}\right)-\frac{t}{2} .
$$

We note that the relations (3.18), (3.19), and (3.20) determine $H_{n}(t)$ within the constant $H_{0}(0)$. This is consistent with the fact that the algebraic variety of the monodromy data is three-dimensional while $w_{n}$ is specified from $w_{0}$ and $w_{1}$. Given the monodromy data $\left\{S_{k}\right\}, H_{0}(0)$ is determined through the relation

$$
e^{2 H_{0}(0)}\left(\Phi_{0,1}^{(\infty)}\right)_{12}=e^{-2 H_{0}(0)}\left(\Phi_{0,1}^{(\infty)}\right)_{21} .
$$

We conclude this section with some comments on the $\tau$-function associated with the solution $w_{n}(t)$. In accordance with the general definition [7], the $\tau$-function corresponding to the nonlinear system (1.8) and (1.9) is determined by

$$
d \ln \tau_{n}=-\frac{1}{2} \operatorname{res}_{z=\infty} \operatorname{Tr} \hat{\Phi}_{n}^{(\infty)}(z)^{-1} \frac{\partial \hat{\Phi}_{n}^{(\infty)}(z)}{\partial z} d T(z),
$$

where, $d T(z)=d\left\{-2 \sigma_{3} z^{4}-2 t z^{2}\right\} \sigma_{3}=-2 z^{2} \sigma_{3} d t$. Using the general recursion equations connecting the coefficients of the formal solution with the coefficients of the corresponding $A$-equation, we find that

$$
\frac{d}{d t} \ln \tau_{n}=-w_{n+1}\left(n+1+w_{n} w_{n+2}\right) \text {. }
$$

Using this equation and the basic equations (1.8) and (1.9) it follows that

$$
\frac{d}{d t} \ln \frac{\tau_{n}}{\tau_{n-1}}=-\left(w_{n+1}+w_{n}\right)
$$

and

$$
\frac{d^{2}}{d t^{2}} \ln \tau_{n}=w_{n+1}\left(w_{n}+w_{n+2}\right)
$$

3.1. An Explicit Solution. The RH problem defined by Eqs. (3.9) and (3.10) can be solved in closed form only if the monodromy data have a special form. In what follows we investigate the case of

$$
S_{2 j}=I, \quad j=1,2,3,4 \text {. }
$$

Since the monodromy data are $n$-independent we consider the case of $n=0$. Now 


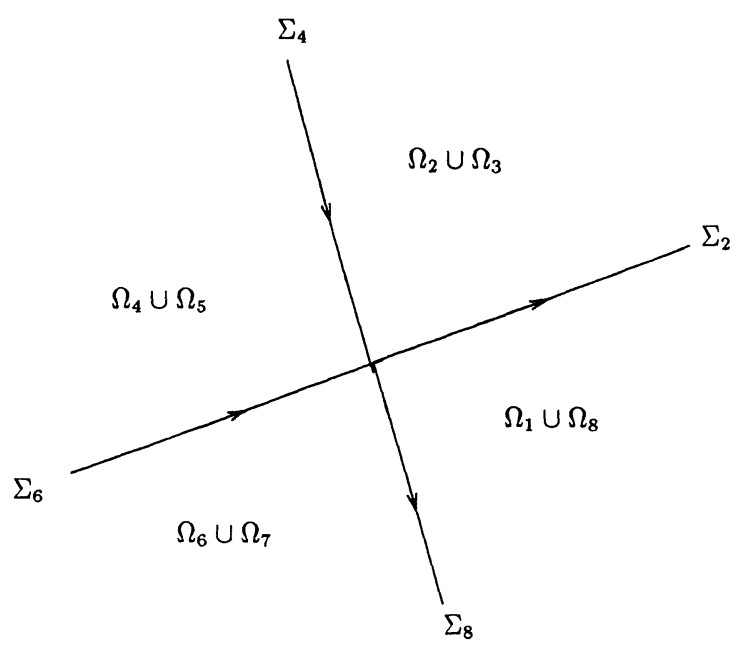

Fig. 3.2

the $\mathrm{RH}$ problem is defined on the contours $\Sigma_{2}, \Sigma_{4}, \Sigma_{6}, \Sigma_{8}$ and

$$
S_{1}=\left(\begin{array}{cc}
1 & s_{1} \\
0 & 0
\end{array}\right), \quad S_{3}=\left(\begin{array}{cc}
1 & s_{3} \\
0 & 1
\end{array}\right), \quad S_{5}=\left(\begin{array}{cc}
1 & -s_{1} \\
0 & 1
\end{array}\right), \quad S_{7}=\left(\begin{array}{cc}
1 & -s_{3} \\
0 & 1
\end{array}\right)
$$

Let's orient the contours $\Sigma_{2}, \Sigma_{4}, \Sigma_{6}$, and $\Sigma_{8}$ as it is shown in Fig. 3.2 and let us introduce the piecewise analytic function $\Phi(z)$ :

$$
\Phi(z) \Lambda_{0} \exp \left\{-2 \sigma_{3}\left(z^{4}+t z^{2}\right)\right\} \equiv\left\{\begin{array}{ll}
\Phi_{0}^{(1)}(z)=\Phi_{0}^{(8)}(z), & z \in \Omega_{1} \cup \Omega_{8} \\
\Phi_{0}^{(2 l+1)}(z)=\Phi_{0}^{(2 l)}(z), & z \in \Omega_{2 l} \cup \Omega_{2 l+1}, \quad l=1,2,3
\end{array} .\right.
$$

The RH-problem under consideration can be reformulated in terms of the function $\Phi(z)$ in the following way:

(a)

$$
\Phi(z)=\left(I+\sum \Phi_{k}^{(\infty)} z^{-k}\right) z^{\sigma_{3}}, \quad z \rightarrow \infty, \quad z \in \Omega_{2 l} \cup \Omega_{2 l+1}, \quad 1 \leqq l \leqq 4, \quad \Omega_{9} \equiv \Omega_{1}
$$

(b) The boundary values $\Phi^{ \pm}(z)$ of the function $\Phi(z)$ on the contours $\sum_{2 l}$ are connected by the relations

where

$$
\Phi^{-}(z)=\Phi^{+}(z) G(z)
$$

$$
\begin{gathered}
G(z)=\left\{\begin{array}{cc}
\left(\begin{array}{cc}
1 & -s_{1} e^{2 \gamma(z)} \\
0 & 1
\end{array}\right), & z \in \Sigma_{2}, \Sigma_{6}, \\
\left(\begin{array}{cc}
1 & s_{3} e^{2 \gamma(z)} \\
0 & 1
\end{array}\right), & z \in \Sigma_{4}, \Sigma_{8},
\end{array}\right. \\
\gamma(z)=H_{0}(t)-2 z^{4}-2 t z^{2} \equiv H_{0}(t)-\frac{1}{2} \bigcup(z) .
\end{gathered}
$$


Using $\Phi_{0,1}^{(\infty)}=\Lambda_{0}^{-1} \Phi_{1}^{(\infty)} \Lambda_{0}$, it follows that

$$
w_{1}=4\left(\Phi_{1}^{(\infty)}\right)_{12}\left(\Phi_{1}^{(\infty)}\right)_{21} .
$$

Let $\Phi=\left(\vec{\Phi}_{1}, \vec{\Phi}_{2}\right)$. The triangularity of the matrix $G(z)$ implies that the vector $\vec{\Phi}_{1}(z)$ is holomorphic everywhere on the $z$-plane, and because of (3.28) it satisfies the following asymptotic condition as $z \rightarrow \infty$ :

$$
\vec{\Phi}_{1}(z) \sim\left(\begin{array}{c}
z+O(1) \\
O(1)
\end{array}\right), \quad z \rightarrow \infty,
$$

This implies the equality

$$
\vec{\Phi}_{1}(z)=\left(\begin{array}{c}
z+d \\
c
\end{array}\right)
$$

where $c, d$ are some constants. The vector $\vec{\Phi}_{2}(z)$ satisfies

$$
\vec{\Phi}_{2}^{-}(z)-\vec{\Phi}_{2}^{+}(z)=\left\{\begin{array}{cc}
-s_{1} e^{2 \gamma(z)}\left(\begin{array}{c}
z+d \\
c
\end{array}\right), & z \in \Sigma_{2}, \Sigma_{6} \\
s_{3} e^{2 \gamma(z)}\left(\begin{array}{c}
z+d \\
c
\end{array}\right), & z \in \Sigma_{4}, \Sigma_{8}
\end{array}\right.
$$

and

$$
\vec{\Phi}_{2}(z) \sim\left(\begin{array}{c}
O\left(\frac{1}{z^{2}}\right) \\
\frac{1}{z}+O\left(\frac{1}{z^{2}}\right)
\end{array}\right), \quad z \rightarrow \infty
$$

Thus

$$
\begin{aligned}
\vec{\Phi}_{2}(z) & =\frac{s_{1} e^{2 H_{0}}}{2 \pi i} \int_{\Sigma_{2} \cup \Sigma_{6}} \frac{e^{-U(\mu)}}{\mu-z}\left(\begin{array}{c}
\mu+d \\
c
\end{array}\right) d \mu-\frac{s_{3} e^{2 H_{0}}}{2 \pi i} \int_{\Sigma_{4} \cup \Sigma_{8}} \frac{e^{-U(\mu)}}{\mu-z}\left(\begin{array}{c}
\mu+d \\
c
\end{array}\right) d \mu \\
& =\frac{s_{1} e^{2 H_{0}}}{2 \pi i} \int_{-\infty}^{\infty} \frac{e^{-U(\mu)}}{\mu-z}\left(\begin{array}{c}
\mu+d \\
c
\end{array}\right) d \mu+\frac{s_{3} e^{2 H_{0}}}{2 \pi i} \int_{-i \infty}^{i \infty} \frac{e^{-U(\mu)}}{\mu-z}\left(\begin{array}{c}
\mu+d \\
c
\end{array}\right) d \mu .
\end{aligned}
$$

The large $z$ asymptotics of the above equation yields

$$
\begin{aligned}
\vec{\Phi}_{2}(z)= & -\frac{1}{z}\left\{\frac{e^{2 H_{0}}}{2 \pi i}\left[s_{1} \int_{-\infty}^{\infty}+s_{3} \int_{-i \infty}^{i \infty}\right] e^{-U(\mu)}\left(\begin{array}{c}
\mu+d \\
c
\end{array}\right) d \mu\right\} \\
& -\frac{1}{z^{2}}\left\{\frac{e^{2 H_{0}}}{2 \pi i}\left[s_{1} \int_{-\infty}^{\infty}+s_{3} \int_{-i \infty}^{i \infty}\right] e^{-U(\mu)} \mu\left(\begin{array}{c}
\mu+d \\
c
\end{array}\right) d \mu\right\}+\cdots
\end{aligned}
$$

Because $U(\mu)$ is an even function, the asymptotic condition (3.33) is satisfied by setting

$$
d=0, \quad c=-\frac{2 \pi i e^{-2 H_{0}}}{s_{1} \int_{-\infty}^{\infty} e^{-U(\mu)} d \mu+s_{3} \int_{-i \infty}^{i \infty} e^{-U(\mu)} d \mu} .
$$


Formulae (3.32), (3.34) and (3.36) give the solution of the RH-problem (3.29). The relations $\left(\Phi_{1}^{(\infty)}\right)_{21}=c,\left(\Phi_{1}^{(\infty)}\right)_{12}=b,\left(\vec{\Phi}_{2}(z)\right)_{1}=\frac{b}{z^{2}}+O\left(\frac{1}{z^{2}}\right), z \rightarrow \infty$, and Eqs.
(3.31), (3.35) and (3.36) imply

$$
\begin{gathered}
w_{1}=4 \frac{s_{1} \int_{-\infty}^{\infty} e^{-U(\mu)} \mu^{2} d \mu+s_{3} \int_{-i \infty}^{i \infty} e^{-U(\mu)} \mu^{2} d \mu}{s_{1} \int_{-\infty}^{\infty} e^{-U(\mu)} d \mu+s_{3} \int_{-i \infty}^{i \infty} e^{-U(\mu)} d \mu} \\
=-\partial_{t} \ln \left\{e^{t^{2} / 2}\left[s_{1} D_{-1 / 2}(\sqrt{2 t})+i s_{3} D_{-1 / 2}(-\sqrt{2 t})\right]\right\},
\end{gathered}
$$

where $D_{v}(y)$ is the parabolic cylinder function.

Note that for the case $n=-1$ the asymptotic behavior of $\Phi(z)$ is

$$
\Phi(z)=\left(I+\sum_{k=1}^{\infty} \Phi^{k} z^{-k}\right), \quad z \rightarrow \infty .
$$

That leads immediately to $\vec{\Phi}_{1}(z)=(1,0)^{T}$ and hence $w_{0}=0$.

Equations (3.37) and $w_{0}=0$ provide and explicit parameterization of the initial data for the solutions of the discrete equation (1.9) with $\gamma=0$ (considered in the domain $n \geqq 0$ ) through the monodromy data in the special case (3.26). For any fixed $n$ the corresponding functions $w_{n}(t)$ are the explicit solutions of the PIV equation (1.10). It should be mentioned that this family of solutions of the PIV has been derived earlier [21] using a similar technique.

\section{The Limit from the Discrete to Continuous Painlevé I}

We first show formally that the transformations

$$
w_{n}=-\frac{t}{3}\left(1-2 h^{2} u(\xi)\right), \quad \frac{n}{t^{2}}=-\frac{1}{3}\left(1+\frac{2}{3} h^{4} \xi\right),
$$

map the discrete PI

$$
n=2 t w_{n}+w_{n}\left(w_{n+1}+w_{n}+w_{n-1}\right)
$$

to the PI equation

$$
\frac{d^{2} u}{d \xi^{2}}=6 u^{2}+\xi
$$

as $h \rightarrow 0$. Indeed, letting $w_{n}=t \rho(1-\varepsilon u)$, where $\rho$ is a constant, Eq. (4.2) becomes as $\varepsilon \rightarrow 0$,

$$
\frac{n}{t^{2} \rho}=2+3 \rho-2(1+3 \rho) \varepsilon u+\rho \varepsilon^{2}\left(3 u^{2}-\frac{h^{2}}{\varepsilon} u_{\xi \xi}\right)
$$

We take $\frac{h^{2}}{\varepsilon}=O(1)$, which we normalize for convenience to be $\frac{1}{2}$, and we take 
$\rho=-1 / 3$ in order to eliminate the $O(\varepsilon)$ term in Eq. (4.4). Then Eq. (4.4) and $w_{n}=t \rho(1-\varepsilon u)$ imply Eqs. (4.1).

The transformation (4.1) with the additional condition

$$
t^{2}=-\frac{9}{2} h^{-5}
$$

also maps the $\mathrm{KM}$ equation (1.8) to

$$
\frac{d^{3} u}{d \xi^{3}}=12 u \frac{d u}{d \xi}+1
$$

as $h \rightarrow 0$. Indeed, letting $w_{n}=-\frac{t}{3}\left(1-2 h^{2} u\right)$ in Eq. (1.8) we find as $h \rightarrow 0$,

$$
2 t h^{2}\left(u_{t}-\frac{2}{3} t h u_{\xi}\right)-\left[1-\frac{2}{9} t^{2} h^{5}\left(u_{\xi \xi \xi}-12 u u_{\xi}\right)\right]=0 .
$$

Equation (4.7) implies Eqs. (4.5), (4.6) and $u_{t}=\frac{2}{3}\left(-\frac{9}{2}\right)^{1 / 2} h^{-3 / 2} u_{\xi}$. This equation is consistent with Eq. (4.1b), since $u_{t}=u_{\xi} \frac{d \xi}{d t}$, and Eq. (4.1b) implies

$$
n t^{-3}=\frac{h^{4}}{9} \frac{d \xi}{d t}, \quad \text { or } \quad \frac{d \xi}{d t}=\frac{9 n}{t^{3} h^{4}} \sim-\frac{3}{t h^{4}}=-3\left(-\frac{9}{2}\right)^{-1 / 2} h^{-3 / 2} .
$$

Remark. In the above derivation we have used the assumption

$$
w_{n \pm 1}(t) \sim \rho t(1-\varepsilon u(\xi \pm h)) \cong \rho t\left(1-\varepsilon u(\xi) \mp \varepsilon u_{\xi}(\xi) h-\frac{\varepsilon h^{2}}{2} u_{\xi \xi}(\xi)\right) .
$$

This assumption is consistent with Eqs. (2.3) iff the equality (4.5) takes place.

In what follows we present a general methodology for:

(a) Showing that the transformations (4.1) and (4.5) in addition to mapping both the KM equation (1.8) and the discrete PI equation (4.2) to PI equation (4.3), they also map the common solutions $w_{n}(t)$ of (4.2) and (1.8), to solutions $u(\xi)$ of (4.3).

(b) Calculating the parameters of the solution $u(\xi)$ in terms of the parameters of the solution $w_{n}(t)$.

This methodology involves the following steps:

(i) Express $w_{n}(t)$ in terms of appropriate monodromy data.

(ii) Express $u(\xi)$ in terms of appropriate monodromy data.

(iii) Study the relationship between the above sets of monodromy data under the limit $h \rightarrow 0$.

Step (i) was presented in Sect. 3. Step (ii), which involves applying the isomonodromy method to solving the PI equation (4.3) was investigated in [22]. Here we only discuss the Lax pair of PI, and we summarize the main results of [22] in Appendix A. The investigation of step (iii) comprises the main subject of this section.

We recall that the Lax pair of the $\mathrm{KdV}$ equation (1.5) is given by

$$
\psi_{x x}+(\Lambda-2 q) \psi=0, \quad \psi_{t}=-2 q_{x} \psi+4(\Lambda+q) \psi_{x} .
$$

The transformations

$$
q(x, t)=-t+u(\xi), \quad \xi=x-6 t^{2}, \quad \lambda=-t-\frac{\Lambda}{2}
$$


map the $\mathrm{KdV}$ equation (1.5) to the PI equation (4.3) and the Lax pairs (4.8) to

$$
\begin{aligned}
\psi_{\xi \xi} & =2(u+\lambda) \psi, \\
\psi_{\lambda} & =2 u_{\xi} \psi+4(2 \lambda-u) \psi_{\xi} .
\end{aligned}
$$

Letting $\vec{\Psi}=\left(\psi, \psi_{\xi}\right)^{T}$, Eqs. (4.10) yield a matrix Lax pair for the matrix eigenfunction $\Psi$. The transformation

$$
\Psi(\xi, \lambda)=(2 \lambda)^{-1 / 4}\left(\begin{array}{cc}
1 & 1 \\
\sqrt{2 \lambda} & -\sqrt{2 \lambda}
\end{array}\right) \sigma_{3} Y(\xi, k) \sigma_{3}, \quad k=(2 \lambda)^{1 / 2},
$$

maps this Lax pair to the one studied in [22]:

$$
\begin{aligned}
& \left.Y_{\xi}=\left[\left(k+\frac{u}{k}\right) \sigma_{3}-\frac{i u}{k} \sigma_{2}\right)\right] Y, \\
& Y_{k}=\left[\left(4 k^{4}+2 u^{2}+\xi\right) \sigma_{3}-i\left(4 u k^{2}+2 u^{2}+\xi\right) \sigma_{2}-\left(2 k u_{\xi}+\frac{1}{2 k}\right) \sigma_{1}\right] Y,
\end{aligned}
$$

where $\sigma_{j}, 1 \leqq j \leqq 3$ are the usual Pauli matrices.

It is straightforward to show formally that under the transformations (4.1), (4.5) and

$$
Q_{n \pm 1} e^{-2 t z^{2}-2 z^{4}}=\psi(\xi \pm h), \quad z=\left(-\frac{t}{3}\right)^{1 / 2}\left(1+h^{2} \lambda\right),
$$

Eqs. (1.2) and (1.3) (with $c_{1}=4 t, c_{2}=2$ ) are mapped to Eqs. (4.10): Consider Eq. (1.2); its right-hand side becomes

$$
\frac{1}{2} w_{n+1}^{1 / 2} Q_{n+1}+\frac{1}{2} w_{n}^{1 / 2} Q_{n-1}=\left(\frac{-t}{3}\right)^{1 / 2}\left(1-h^{2} u\right)\left(\psi+\frac{h^{2}}{2} \psi_{\xi \xi}\right) e^{2 t z^{2}+2 z^{4}} .
$$

Comparing this with the left-hand side of (1.2) it follows that $z$ is given by (4.13b). Using (1.2), Eq. (1.3) can be represented in the following equivalent form:

$$
Q_{n_{z}}=-4 Q_{n} z w_{n}+Q_{n-1}\left(4 t w_{n}^{1 / 2}+2 w_{n}^{1 / 2}\left(w_{n}+w_{n+1}\right)+8 z^{2} w_{n}^{1 / 2}\right) .
$$

Using Eqs. (4.1), (4.5) and (4.13) we find the relation

$$
h^{3} \psi_{\lambda} \cong \psi\left(8 h^{2} \lambda-4 h^{2} u\right)+\left(\psi-h \psi_{\xi}\right)\left(4 h^{2} u-8 h^{2} \lambda+2 h^{3} u_{\xi}\right), \quad h \rightarrow 0,
$$

which immediately yields the second equation in (4.10).

Recalling that $\vec{\Phi}_{n}=\left(Q_{n+1}, Q_{n}\right)^{T} \exp \left(-2 t z^{2}-2 z^{4}\right)$ (Eq. (3.1)) and $\overrightarrow{\boldsymbol{\Psi}}=\left(\psi, \psi_{\xi}\right)^{T}$, the above calculation implies

$$
\Phi_{n-1}=\left(\begin{array}{cc}
1 & 0 \\
1 & -h
\end{array}\right) \Psi, \quad \text { as } \quad h \rightarrow 0 .
$$

We note that Eq. (4.13b) is not well defined because of the multivalueness of the right-hand side. The precise meaning of Eq. (4.13b) will be elucidated later.

4.1. The Connection between Monodromy Data of the Discrete and Continuous PI Equations with $t$ Imaginary. The formula (4.1) and the exponential term in (3.8) 
suggest the scaling

$$
t=i \tau, \quad \tau>0, \quad z=\sqrt{\tau} \zeta .
$$

Then our formal results above can be summarized as follows: The transformations

$$
\tau^{2}=\frac{9}{2} h^{-5}, \quad \frac{n}{\tau^{2}}=\frac{1}{3}\left(1+\frac{2}{3} h^{4} \xi\right), \quad w_{n}(\tau)=-\frac{i \tau}{3}\left(1-2 h^{2} u(\xi)\right), \quad h \rightarrow 0
$$

map both the KM equation (1.8) and the discrete PI equation (4.2), to the continuous PI equation (4.3). Furthermore, if in addition

$$
\zeta=\frac{ \pm e^{-i \pi / 4}}{\sqrt{3}}\left(1+\frac{h^{2} k^{2}}{2}\right), \quad \Phi_{n-1}(\tau, \zeta)=k^{-1 / 2}\left(\begin{array}{cc}
1 & 1 \\
1-h k & 1+h k
\end{array}\right) \sigma_{3} Y(\xi, k) \sigma_{3},
$$

then the discrete Lax pairs (3.2)-(3.4) are mapped to the Lax pair (4.12) of PI.

Since the solution $w_{n}(\tau)$ of $(1.8)$ and of (4.2) is basically determined by Eq. (3.4) we concentrate on this equation. It is more convenient to work. with $\Phi_{n-1}$ instead of $\Phi_{n}$; using $t=i \tau, z=\sqrt{\tau} \zeta$ it follows that

where

$$
\frac{\partial}{\partial \zeta} \Phi_{n-1}=\tau^{2} B_{n-1} \Phi_{n-1}
$$

$$
B_{n}=\left(\begin{array}{cc}
a_{n} & b_{n} \\
c_{n} & -a_{n}
\end{array}\right), \begin{aligned}
& a_{n}=-8 \zeta^{3}-4 i \zeta-4 \zeta \tau^{-1} w_{n+1} \\
& b_{n}=2 \tau^{-3 / 2} w_{n+1}^{1 / 2}\left(2 i \tau+4 \tau \zeta^{2}+w_{n+1}+w_{n+2}\right) \\
& c_{n}=-2 \tau^{-3 / 2} w_{n+1}^{1 / 2}\left(2 i \tau+4 \tau \zeta^{2}+w_{n}+w_{n+1}\right)
\end{aligned}
$$

Equation (4.18) with $\tau$ large, can be solved by the WKB method. Diagonalizing the matrix $B_{n-1}$ we find

$$
T_{n-1}^{-1} B_{n-1} T_{n-1}=-\mu \sigma_{3} ; \quad \mu=\sqrt{-\operatorname{det} B_{n-1}}, \quad T_{n-1}=\left(\begin{array}{cc}
1 & 1 \\
-\frac{\mu+a_{n-1}}{b_{n-1}} & \frac{\mu-a_{n-1}}{b_{n-1}}
\end{array}\right) .
$$

The turning points $\zeta_{T}$ are given by

$$
\mu\left(\zeta_{T}\right)=0=\left[64\left(\zeta^{2}+\frac{i}{3}\right)^{3}+O\left(h^{4}\right)\right]^{1 / 2},
$$

i.e. there exist two turning points of third order,

$$
\zeta_{ \pm}= \pm \frac{e^{-i \pi / 4}}{\sqrt{3}}
$$

In the rest of this section we concentrate on one of the turning points. Using (4.20), it follows that the WKB solution of (4.18) is given by

$$
\Phi_{n-1}^{\mathrm{WKB}} \sim T_{n-1}(\zeta) \exp \left\{-\tau^{2} \sigma_{3} \int_{\zeta_{+}}^{\zeta} \mu\left(\zeta^{\prime}\right) d \zeta^{\prime}-\int^{\zeta} \operatorname{diag}\left(T_{n-1}^{-1}\left(\zeta^{\prime}\right) d \zeta^{\prime} T_{n-1}\left(\zeta^{\prime}\right)\right) d \zeta^{\prime}\right\},
$$


or

$$
\begin{gathered}
\Phi_{n-1}^{\mathrm{WKB}} \sim\left(\zeta^{2}-\zeta_{+}^{2}\right)^{-1 / 4}\left[\left(\begin{array}{cc}
m^{1 / 2} & m^{-1 / 2} \\
m^{-1 / 2} & m^{1 / 2}
\end{array}\right)+O\left(\frac{h^{2}}{\left(\zeta^{2}-\zeta_{+}^{2}\right)}\right)\right] \\
\cdot \exp \left\{-\tau^{2} \sigma_{3} \int_{\zeta_{+}}^{\zeta} \mu\left(\zeta^{\prime}\right) d \zeta^{\prime}\right\},
\end{gathered}
$$

where $m \doteqdot\left[\zeta+\left(\zeta^{2}-\zeta_{+}^{2}\right)^{1 / 2}\right] / \zeta_{+}$. The Stokes lines of the WKB solution are given by $\operatorname{Re} \int_{\zeta_{ \pm}}^{\zeta} \mu(\zeta) d \zeta=0$. Therefore WKB solutions about $\zeta_{+}$and $\zeta_{-}$can be used for the evaluation of the solutions $\Phi_{n-1}^{(1)}, \Phi_{n-1}^{(2)}, \Phi_{n-1}^{(7)}, \Phi_{n-1}^{(8)}$ and $\Phi_{n-1}^{(3)}, \Phi_{n-1}^{(3+j)}, 0 \leqq j \leqq 3$ respectively (see Fig. 4.1). Hence,

$$
\begin{aligned}
\Phi_{n-1}^{(j)} & ={\stackrel{\operatorname{WKB}}{\Phi_{n-1}(J)}}_{\Lambda_{n-1}}\left(\frac{\zeta_{+}}{2}\right)^{1 / 2} e^{\sigma_{3}\left(\delta_{x}+(n / 2) \ln \tau\right)}, \\
\delta_{\infty} & \doteqdot \lim _{\zeta \rightarrow \infty}\left[\tau^{2} \int_{\zeta_{+}}^{\zeta} \mu\left(\zeta^{\prime}\right) d \zeta^{\prime}-2 \tau^{2} \zeta^{4}-2 i \tau^{2} \zeta^{2}+n \ln \zeta\right],
\end{aligned}
$$

where $j=1,2,7,8$.

In the neighborhood of the turning point $\zeta_{+}$, the WKB asymptotics (4.22) breaks down. In this neighborhood we introduce the new variable $k$ by (see Eq. (4.17a))

$$
\zeta=\zeta+\left(1+\frac{h^{2} k^{2}}{2}\right)
$$

Then Eq. (4.17b) indicates that the solution $\Phi_{n-1}$ at the turning point $\zeta_{+}$, which we denote by $\Phi_{n-1}$, is related to the solution $Y(\xi, k)$ associated with PI. More precisely

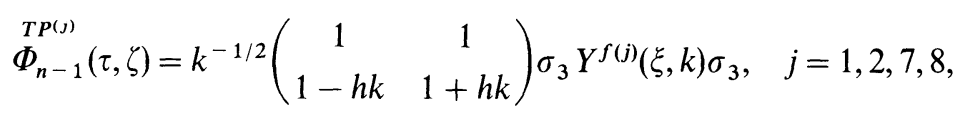

where $f(1)=1, f(2)=2, f(7)=-1, f(8)=0$ (see Fig. 4.1).

Our aim is to relate the monodromy data $\{S\}$ associated with $\Phi_{n-1}$ to the monodromy data $\left\{G_{\mathrm{WKB}^{(j)}}\right\}_{T^{(j)}}$ associated with $Y$. This is now straightforward, since we can relate $\Phi_{n-1}$ to $\Phi_{n-1}$ and hence we can relate $\Phi_{n-1}^{(j)}$ to $Y^{f(j)}$ : In the matching domain $\frac{k^{2}}{2} \sim h^{-\varepsilon}, \varepsilon>0$,

$$
\begin{aligned}
\mathscr{\Phi}_{n-1}^{T(\jmath)} & ={ }_{n-1}^{\operatorname{WKB}(\jmath)}\left(h \zeta_{+}\right)^{1 / 2} e^{\delta+\sigma_{3}(1+o(1))}, \\
\delta_{+} & =\lim _{k \rightarrow \infty}\left[-\int_{0}^{k^{2}}\left(4 k^{\prime 3}+2 \xi k^{\prime}+r\right)^{1 / 2} d k^{\prime}+\frac{4}{5} k^{5}+\xi_{k}\right],
\end{aligned}
$$

where $r=u_{\xi}^{2}-4 u^{3}-2 \xi u$. To obtain this equation we use the fact that in the 


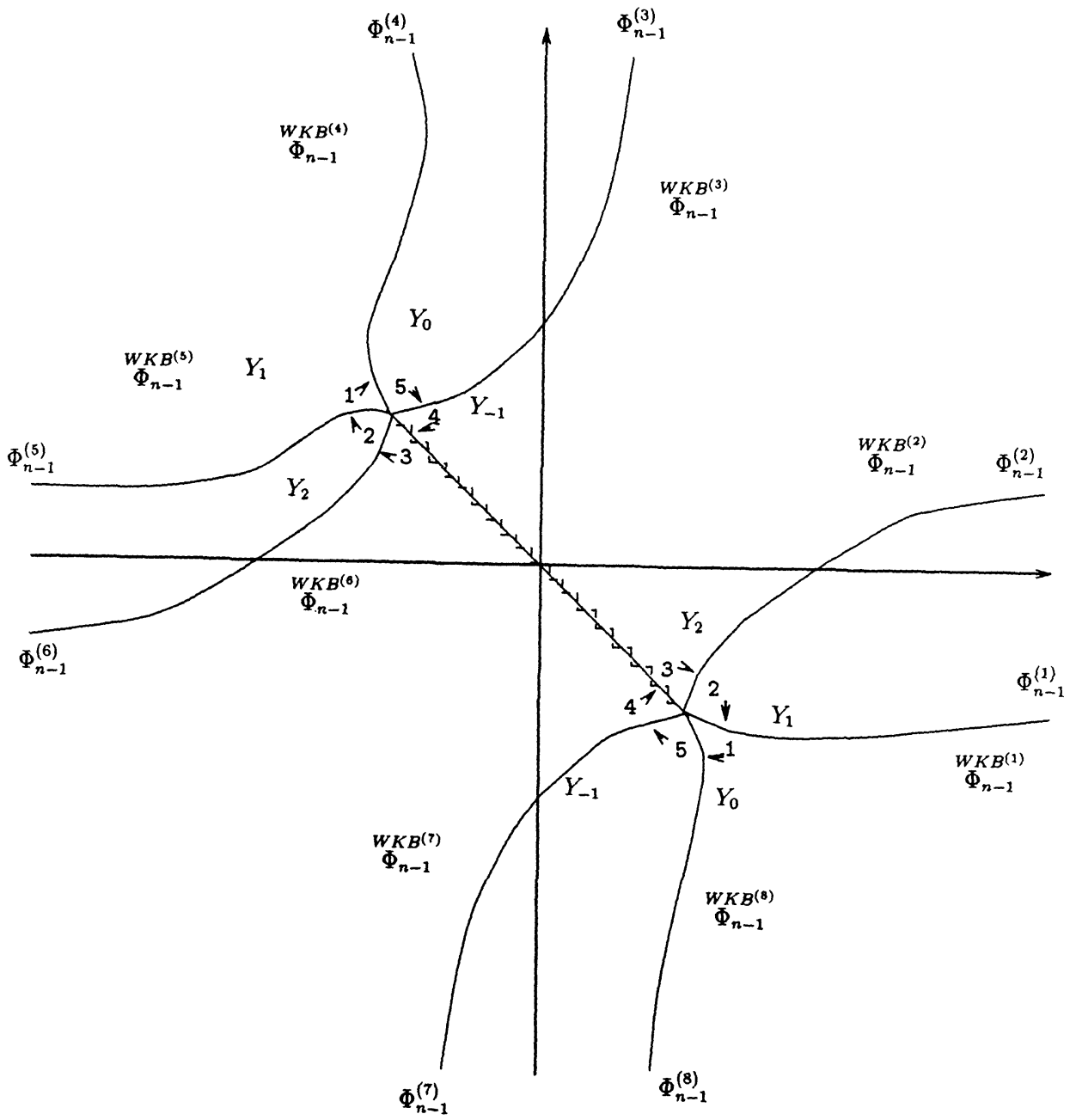

RAY 1: $\arg k=-\frac{\pi}{10}$, RAY 2: $\operatorname{argk}=\frac{\pi}{10}$,

RAY 3: $\arg k=\frac{3 \pi}{10}$, RAY 5: $\arg k=-\frac{3 \pi}{10}$

Fig. 4.1

matching domain

$$
-\tau^{2} \int_{\zeta_{+}}^{\zeta} \mu\left(\zeta^{\prime}\right) d \zeta^{\prime} \sim 2 \int_{0}^{\lambda}\left(32 \lambda^{\prime 3}+4 \xi \lambda^{\prime}+r\right)^{1 / 2} d \lambda^{\prime} .
$$

Using Eqs. (4.23), (4.25), and (4.26) and the definition of the monodromy data

$$
S_{j}=\left(\Phi_{n-1}^{(j)}\right)^{-1} \Phi_{n-1}^{(j+1)}, \quad G_{f(j)}=\left(Y^{f(j)}\right)^{-1} Y^{f(j)+1}
$$

we find

$$
S_{j} \cong e^{\alpha \sigma_{3}} \sigma_{3} G_{f(j)} \sigma_{3} e^{-\alpha \sigma_{3}}, \quad j=1,7,8 ; \quad \alpha=\delta_{+}(\xi)-\delta_{\infty}(n, \tau)-\frac{n}{2} \ln \tau-H_{n-1}(\tau) .
$$


Recalling that $S_{j}$ are independent of $n$ and of $t$ we obtain the main result of this section:

$$
\begin{gathered}
S_{j}=e^{\alpha_{0} \sigma_{3}} \sigma_{3} G_{f(j)} \sigma_{3} e^{-\alpha_{0} \sigma_{3}}, \quad \alpha_{0}=\lim _{h \rightarrow 0}\left(\delta_{+}(\xi)-\delta_{\infty}(n, \tau)-\frac{n}{2} \ln \tau-H_{n-1}(\tau)\right) \\
j=1,7,8
\end{gathered}
$$
Evaluating the integral $\int_{\xi_{+}}^{\xi} \mu(\xi) d \xi$ under the asymptotic condition (4.16) it
follows that

$$
\delta_{\infty}(n, \tau)=-\frac{\tau^{2}}{4}-n \ln 2 \sqrt{3}-\delta_{+}(\xi)-\frac{i \pi n}{4}+o(1), \quad h \rightarrow 0 .
$$

This means that we have also obtained the asymptotic formula for the quantity $H_{n}(t)$ :

$$
H_{n-1}(t)=-\frac{t^{2}}{4}-\frac{n}{2} \ln t+n \ln 2 \xi_{+}+2 \delta_{+}(\xi)-\alpha_{0}+o(1) .
$$

This equality is formally consistent with Eq. (3.18). It is interesting to note that it does not appear possible to obtain equality (4.30) directly from Eqs. (3.18) or (3.19) using Eq. (4.16) only.

The manifold of the monodromy data $\{G\}$ is two-dimensional (see Appendix A). The formula (4.28) shows that the monodromy data $\{G\}$ depends on the orbits (3.17) only; the constant $\alpha_{0}$ parameterizes the transversal directions of the manifold $\{S\}$.

4.2. The Triangular Case. In this special case considered in Sect. 3.2, the monodromy data are given by (3.26) and (3.27). Then Eq. (4.29) yields

$$
g_{5}=0, \quad \frac{g_{1}}{g_{4}}=-\frac{s_{1}}{s_{3}} .
$$

This corresponds to the case (A.5) of Appendix A, and the corresponding solution $u(\xi)$ of the equation PI (4.3) can be characterized by one of the asymptotic formulae (A.7-A.9) with

$$
g_{1}=\frac{i}{1+p}, \quad g_{4}=\frac{i p}{1+p}, \quad p=-\frac{s_{3}}{s_{1}} .
$$

Equations (4.32) indicate that $p \neq-1$ is a necessary condition of the existence of the limit (4.1), (4.5) of the solution $w_{n}(t)$. For the constant $\alpha_{0}$ in (4.30) we have from (4.29) and (4.32),

$$
\alpha_{0}=\frac{1}{2} \ln \frac{i s_{1}^{2}}{s_{3}-s_{1}}
$$

We emphasizes that the results obtained in this section are formal: Assuming that the solution $w_{n}(t)$ has the asymptotic behavior (4.16) we have derived the formula (4.29) connecting the monodromy of $w_{n}(t)$ and of $u(\xi)$. This means that the solvability of the algebraic equation (4.29) is a necessary condition of the 
existence of the limit (4.16) for given $\{S\}$. Our previous experience with the isomonodromic approach suggests that the solvability of the Eq. (4.29) is also a sufficient condition. Rigorous aspects of these results are under investigation.

\section{An Application in the Theory of 2D Quantum Gravity}

It turns out that there exists an intimate connection between the special solutions of Eqs. (1.8) and (1.9) studied in Sects. 3.2 and 4.2 and the matrix model in the theory of $2 \mathrm{D}$ quantum gravity. The partition function of this model is defined by the equality

$$
Z_{n}(\vec{t})=\int d \Phi \exp \{-\operatorname{Tr} U(\Phi)\}
$$

Here $\Phi$ is a $n \times n$-hermitian matrix, and the potential $U(z)$ is an arbitrary even function of $z$ depending parametrically on $\vec{t} \equiv\left(t_{1}, t_{2}, t_{3}, \ldots\right)$. As it is shown in [26], it is possible to represent $Z_{n}$ in the form

$$
Z_{n}=\prod_{j=1}^{n} h_{j}
$$

where $h_{n}$ are the volume elements associated with the orthogonal polynomials $P_{n}(z)$ with respect of the measure $d \mu(z) \doteqdot \exp (-U(z)) d z$ :

$$
\delta_{n m} h_{n}=\int_{-\infty}^{\infty} P_{n}(z) P_{m}(z) d \mu(z), \quad P_{n}=z^{n}+\cdots .
$$

Note that $h_{n}$ and $P_{n}$ depend parametrically on $t_{1}, t_{2}, \ldots$, but for convenience of notation we suppress this dependence. Equation (5.2) means that the question of computing the partition function (5.1) reduces to the question of evaluating $h_{n}$. Following ideas of [10 and 11] we first show that:

(a) $P_{n}$ satisfies the recurrence relationship

$$
z P_{n}=P_{n+1}+\frac{w_{n}}{4} P_{n-1}, \quad w_{n} \doteqdot 4 \frac{h_{n}}{h_{n-1}} .
$$

This becomes Eq. (1.2), if $Q_{n} \doteqdot P_{n} / h_{n}^{1 / 2}$.

(b) $h_{n}$ satisfies the spatially discrete evolution equation

$$
\frac{\partial h_{n}}{\partial t_{k}}=-\int_{-\infty}^{\infty} P_{n}^{2}(z) \frac{\partial U}{\partial t_{k}} d \mu(z), \quad k=1,2, \ldots,
$$

and the discrete Painlevé equation

$$
(1+2 n) h_{n}=\int_{-\infty}^{\infty} z P_{n}^{2}(z) U^{\prime}(z) d \mu(z) .
$$

One can use the recurrence relation (5.4) to eliminate the powers of the variable $z$ from the right-hand side of both $(5.5)^{k}$ and (5.6) and to obtain nonlinear difference equations for $h_{n}$. An elegant way of achieving this will be given later in this section.

(c) Equations (5.5) $)^{k}$ and (5.6) are integrable, in the sense that they admit a Lax 
pair formulation. The Lax pair of Eq. (5.5) is Eq. (5.4) together with

$$
P_{n_{t}}=\sum_{m=0}^{n} C_{n m} P_{m}, \quad C_{n m}=\frac{1}{h_{m}} \int_{-\infty}^{\infty} P_{n} P_{m} \frac{\partial U}{\partial t_{k}} d \mu, \quad C_{n n}=\frac{1}{2} \partial_{t_{k}} \ln h_{n}+\frac{1}{2 h_{n}} \int_{-\infty}^{\infty} P_{n}^{2} \frac{\partial U}{\partial t_{k}} d \mu
$$

while, the Lax pair of Eq. (5.6) is Eq. (5.4) together with

$$
P_{n_{z}}=\sum_{m=0}^{n-1} D_{n m} P_{m}, \quad D_{n m}=\frac{1}{h_{m}} \int_{-\infty}^{\infty} P_{n} P_{m} \frac{\partial U}{\partial z} d \mu .
$$

We now derive the above results. In order to derive the recurrence relationship (5.4) we note that the orthogonal polynomials $P_{n}$ satisfy the symmetry condition

$$
P_{n}(-z)=(-1)^{n} P_{n}(z) \text {. }
$$

Indeed, using the fact that $U(z)$ is even, it follows that Eq. (5.3) remain invariant under the transformation (5.9). Any polynomial from the orthogonal set $\left\{P_{n}\right\}$ is orthogonal to any other polynomial with lower degree. Thus,

$$
{ }_{z} P_{n}=A_{n} P_{n+1}+B_{n} P_{n}+C_{n} P_{n-1} .
$$

The normalizing condition (5.3b) implies $A_{n}=1$. Furthermore,

$$
B_{n} h_{n}=\int_{-\infty}^{\infty} z P_{n}^{2}(z) d \mu(z)
$$

using Eq. (5.9) it follows that $B_{n}=0$. Also

$$
C_{n} h_{n-1}=\int_{-\infty}^{\infty} z P_{n} P_{n-1} d \mu(z)=\int_{-\infty}^{\infty} P_{n}\left(P_{n}+\cdots\right) d \mu(z)=h_{n},
$$

where the dots mean polynomials of degree lower than $n$. Therefore, the recurrence relationship for the orthogonal polynomials defined by an arbitrary even measure is Eq. (5.4). To derive Eq. $(5.5)^{k}$ we note that $\operatorname{deg} \frac{\partial P}{\partial t_{k}}$ is less than $n$, thus $\frac{\partial P_{n}}{\partial t_{k}}$ is orthogonal
to $P_{n}$. Then Eq. (5.3) implies $(5.5)^{k}$.

To derive Eq. (5.6) we use integration by parts:

$$
h_{n}=\int_{-\infty}^{\infty} P_{n}^{2} e^{-U} \cdot 1 d z=-\int_{-\infty}^{\infty} z\left[2 P_{n} P_{n}^{\prime}-P_{n}^{2} U^{\prime}\right] d \mu(z)
$$

But

$$
\int_{-\infty}^{\infty} z P_{n} P_{n}^{\prime} d \mu(z)=n \int_{-\infty}^{\infty} P_{n}\left(P_{n}+\cdots\right) d \mu(z)=n h_{n} .
$$

Thus Eq. (5.10) becomes Eq. (5.6) ${ }^{n}$

To derive Eq. (5.7) we let $P_{n_{t_{k}}}=\sum_{m=0}^{n} C_{n m} P_{m}$, hence

$$
h_{m} C_{n m}=\int_{-\infty}^{\infty} P_{m} P_{n_{t_{k}}} d \mu, \quad m \leqq n .
$$


But Eq. (5.3) implies

$$
O=\int_{-\infty}^{\infty} P_{n_{t_{k}}} P_{m} d \mu+\int_{-\infty}^{\infty} P_{n} P_{m_{t_{k}}} d \mu-\int_{-\infty}^{\infty} P_{n} P_{m} U_{t_{k}} d \mu, \quad m \neq n
$$

and

$$
h_{n_{t_{k}}}=\int_{-\infty}^{\infty} 2 P_{n} P_{n_{t_{k}}} d \mu-\int_{-\infty}^{\infty} P_{n}^{2} U_{t_{k}} d \mu .
$$

Using the fact that $\operatorname{deg} P_{m_{t_{k}}}<m$ and $m \leqq n$, the second term in Eq. (5.12) is zero. Solving for $\int_{-\infty}^{\infty} P_{m} P_{n_{t_{k}}} d \mu$ from Eqs. (5.12) and (5.13) and using (5.10), we find
Eq. (5.7).

To derive Eq. (5.8) we let $P_{n_{z}}=\sum_{m=0}^{n} D_{n m} P_{m}$, hence

$$
h_{n} D_{n m}=\int_{-\infty}^{\infty} P_{m} P_{n_{z}} d \mu
$$

and using (5.3) we find $D_{n n}=0$ and $h_{n} D_{n m}=\int_{-\infty}^{\infty} P_{n} P_{m} U^{\prime} d \mu$.

It is convenient to write the basic equations (5.4)-(5.8) in terms of the variable $Q$. Also we consider a special form $U$. Using

$$
Q_{n} \doteqdot \frac{P_{n}}{h_{n}^{1 / 2}}, \quad U \doteqdot \sum_{k=1}^{N} t_{k} z^{2 k}
$$

Eqs. (5.4)-(5.8) become Eq. (1.2) and equations

$$
\begin{gathered}
\partial_{t k} \ln h_{n}=-\int_{-\infty}^{\infty} Q_{n}^{2} z^{2 k} d \mu, \text { or } \partial_{t_{k}} \ln w_{n}=\int_{-\infty}^{\infty}\left(Q_{n-1}^{2}-Q_{n}^{2}\right) z^{2 k} d \mu, \\
1+2 n=\sum_{k=1}^{N} 2 k t_{k} \int_{-\infty}^{\infty} Q_{n}^{2} z^{2 k} d \mu, \\
Q_{n_{t_{k}}}=\sum_{m=0}^{n-1}\left(\int_{-\infty}^{\infty} Q_{n} Q_{m} z^{2 k} d \mu\right) Q_{m}+\left(\frac{1}{2} \int_{-\infty}^{\infty} Q_{n}^{2} z^{2 k}\right) Q_{n}, \\
Q_{n_{z}}=\sum_{m=0}^{n-1}\left(\sum_{k=1}^{N} 2 k t_{k} \int_{-\infty}^{\infty} Q_{n} Q_{m} z^{2 k-1} d \mu\right) Q_{m} .
\end{gathered}
$$

It is possible to represent Eqs. (1.2) and (5.15)-(5.18) in an elegant form using a notation similar to the one introduced in [10 and 11]. Let $H$ be the space of sequences $Q=\left\{Q_{n}\right\}_{n=-\infty}^{n=\infty}$; for any operator $M$ in $H$ we can define its matrix elements $M_{n m}$ through the equality

$$
(M Q)_{n}=\sum_{m=-\infty}^{\infty} M_{n, m} Q_{m} .
$$

In the space $H$ we introduce the operator

$$
L \doteqdot \frac{1}{2} \Delta w^{1 / 2}+\frac{1}{2} w^{1 / 2} \Delta^{-1}
$$


where the operators $\Delta$ and $\omega$ are defined by

$$
(w Q)_{n}=w_{n} Q_{n}, \quad(\Delta Q)_{n}=Q_{n+1} .
$$

Then Eq. (1.2) takes the form of an eigenvalue equation for the operator $L$,

$$
L Q=z Q \text {. }
$$

We note that the matrix elements of the operator $L$ are given by

$$
L_{n, m}=\frac{1}{2} w_{m}^{1 / 2} \delta_{n+1, m}+\frac{1}{2} w_{n}^{1 / 2} \delta_{n-1, m},
$$

and $L_{n, m}=L_{m, n}$. It is straightforward to compute the matrix elements of $L^{k}$. For example

$$
4 L^{2}=\Delta w^{1 / 2} \Delta w^{1 / 2}+w+\Delta w \Delta^{-1}+w^{1 / 2} \Delta^{-1} w^{1 / 2} \Delta^{-1}
$$

and

$$
4 L_{n, m}^{2}=w_{m-1}^{1 / 2} w_{m}^{1 / 2} \delta_{n+2, m}+w_{n}+w_{n+1}+w_{n}^{1 / 2} w_{m+1}^{1 / 2} \delta_{n+2, m}
$$

in general $\Delta^{j}$ gives rise to $\delta_{n+j, m}$.

For the case of orthogonal polynomials we restrict Eq. (5.21) to the subspace $H_{+}=\left\{Q \in H, Q_{n}=0\right.$ for $\left.n<0\right\}$. This implies the condition

$$
w_{0}=0 \text {, }
$$

in order for $H_{+}$and $H_{-}=H \ominus H_{+}$to be invariant for $L$. Then

$$
\begin{aligned}
\int_{-\infty}^{\infty} Q_{n}(z) Q_{m}(z) z^{2 k} d \mu(z) & =\int_{-\infty}^{\infty} Q_{m}(z)\left(L^{2 k} Q(z)\right)_{n} d \mu(z) \\
& =\sum_{l=0}^{\infty} L_{n, l}^{2 k} \int_{-\infty}^{\infty} Q_{m}(z) Q_{l}(z) d \mu(z)=\sum_{l=0}^{\infty} L_{n, l}^{2 k} \delta_{m l}=L_{n, m}^{2 k} .
\end{aligned}
$$

Using this relationship, Eqs. (5.15)-(5.18) become

$$
\begin{gathered}
\partial_{t_{k}} \ln h_{n}=-L_{n n}^{2 k}, \quad \text { or } \quad \partial_{t k} \ln w_{n}=L_{n-1, n-1}^{2 k}-L_{n, n}^{2 k}, \\
1+2 n=\sum_{k=1}^{N} 2 k t_{k} L_{n n}^{2 k}, \\
Q_{t k}=\left(L_{-}^{2 k}+\frac{1}{2} L_{0}^{2 k}\right) Q, \\
Q_{z}=\sum_{k=1}^{N-1} 2 k t_{k} L_{-}^{2 k-1} Q,
\end{gathered}
$$

where we have used the decomposition

$$
L^{2 k}=L_{+}^{2 k}+L_{0}^{2 k}+L_{-}^{2 k}
$$

for the positive, zero, and negative with respect to $\Delta$ parts.

Example. $N=2$. Equation (5.23) indicates that $L_{n, n}^{2}=\frac{1}{4}\left(w_{n}+w_{n+1}\right)$, thus Eq. (5.25) with $k=1$ becomes

$$
\partial_{t_{1}} \ln h_{n}=-\frac{1}{4}\left(w_{n}+w_{n+1}\right) \quad \text { or } \quad \partial_{t_{1}} \ln w_{n}=\frac{1}{4}\left(w_{n-1}-w_{n+1}\right) .
$$


Also,

$$
\begin{aligned}
8 L^{3}= & \Delta w^{1 / 2} \Delta w^{1 / 2} \Delta w^{1 / 2}+\Delta w^{3 / 2}+\Delta w^{1 / 2} \Delta w \Delta^{-1}+w \Delta w^{1 / 2} \\
& +\Delta w \Delta^{-1} w^{1 / 2} \Delta^{-1}+w^{1 / 2} \Delta^{-1} w+w^{3 / 2} \Delta^{-1}+w^{1 / 2} \Delta^{-1} w^{1 / 2} \Delta^{-1} w^{1 / 2} \Delta^{-1}
\end{aligned}
$$

thus

$$
\begin{aligned}
16 L_{0}^{4}= & \Delta w^{1 / 2} \Delta w \Delta^{-1} w^{1 / 2} \Delta^{-1}+\Delta w \Delta^{-1} w \\
& +\Delta w^{2} \Delta^{-1}+w^{2}+w \Delta w \Delta^{-1}+w^{1 / 2} \Delta^{-1} w \Delta w^{1 / 2}
\end{aligned}
$$

Hence, Eq. (5.26) yields

$$
1+2 n=I_{n}+I_{n+1}, \quad I_{n} \doteqdot \frac{t_{1}}{2} w_{n}+\frac{t_{2}}{4} w_{n}\left(w_{n+1}+w_{n}+w_{n-1}\right) .
$$

Using the explicit forms of $L_{-}$and $L_{-}^{3}$, Eq. (5.28) becomes

$$
Q_{n_{z}}=\left[t_{1} w_{n}^{1 / 2}+\frac{t_{2}}{2} w_{n}^{1 / 2}\left(w_{n+1}+w_{n-1}+w_{n}\right)\right] Q_{n-1}+\frac{t_{2}}{2}\left(w_{n} w_{n-1} w_{n-2}\right)^{1 / 2} Q_{n-3} .
$$

Letting $t_{1}=4 t, t_{2}=4$, Eqs. (5.30) and (5.32) become Eqs. (1.8) and (1.3) respectively; also Eq. (1.9) is the integrated form of (5.31) (we use $w_{0}=0$ to evaluate the constant of "integration"). Similarly, Eq. (5.27) is Eq. (2.1).

The compatibility condition of the linear equations (5.21) and (5.27) and of (5.21) and (5.28) implies the Lax-type equations

$$
L_{t_{k}}=\left[L_{-}^{2 k}+\frac{1}{2} L_{0}^{2 k}, L\right]
$$

and

$$
\left[L, U_{-}^{\prime}(L)\right]=1,
$$

where we have used $U(z)=\sum_{k=0}^{N} t_{k} z^{k}$. Actually it can be shown that Eqs. (5.33) and (5.34) coincide with Eqs. (5.25) and (5.26) respectively. The proof of this statement is given in Appendix B. Here we check this fact explicitly for the case $N=2, k=1$ : Using the explicit forms of the relevant operators we find,

$$
\begin{aligned}
{\left[L_{-}^{2}+\frac{1}{2} L_{0}^{2}, L\right]=} & \frac{1}{16} w^{1 / 2} \Delta^{-1} w+\frac{1}{16} w \Delta w^{1 / 2} \\
& -\frac{1}{16} \Delta w \Delta^{-1} w^{1 / 2} \Delta^{-1}-\frac{1}{16} \Delta w^{1 / 2} \Delta w \Delta^{-1} \\
{\left[L, U_{-}^{\prime}(L)\right]=\frac{t_{1}}{2} \Delta w \Delta^{-1}+} & \frac{t_{2}}{4}\left(\Delta w \Delta^{-1} w+\Delta w^{2} \Delta^{-1}+\Delta w^{1 / 2} \Delta w \Delta^{-1} w^{1 / 2} \Delta^{-1}\right) \\
& -\frac{t_{1}}{2} w-\frac{t_{2}}{4}\left(w^{1 / 2} \Delta^{-1} w \Delta w^{1 / 2}+w^{2}+\Delta w \Delta^{-1} w\right) .
\end{aligned}
$$

Thus, the nontrivial elements of the above commutators are

$$
\begin{aligned}
{\left[L_{-}^{2}+\frac{1}{2} L_{0}^{2}, L\right]_{n, n+1} } & =\frac{1}{16} w_{n+1}^{1 / 2}\left(w_{n}-w_{n+2}\right), \\
{\left[L_{-}^{2}+\frac{1}{2} L_{0}^{2}, L\right]_{n, n-1} } & =\frac{1}{16} w_{n}^{1 / 2}\left(w_{n-1}-w_{n+1}\right), \\
{\left[L, U_{-}^{\prime}(L)\right]_{n, n} } & =I_{n+1}-I_{n} .
\end{aligned}
$$


The above equations and Eqs. (5.33), (5.34) imply Eq. (5.30) and $I_{n+1}-I_{n}=1$, or $I_{n}=n$ (using again that $w_{0}=0$ ).

The results obtained above show that the partition function $Z_{n}(\vec{t})$ can be represented as

$$
\ln Z_{n}=\sum_{j=1}^{n}(n-j+1) \ln \frac{w_{j}}{4},
$$

where $w_{n}(\vec{t})$ is the common solution of the commuting Lax-type equations (5.33). It should be emphasized that this $w_{n}$ is the special self-similar solution of (5.33) satisfying simultaneously the discrete Painlevé equation (5.34) supplemented with the initial data

$$
w_{0}=0, \quad w_{j}=\frac{4 h_{j}}{h_{j-1}}, \quad j=1,2, \ldots, N-1 .
$$

Here, $h_{j}$ are determined successively through (5.3): For instance,

$$
h_{0}=\int_{-\infty}^{\infty} e^{-U(z)} d z, \quad h_{1}=\int_{-\infty}^{\infty} z^{2} e^{-U(z)} d z, \quad h_{2}=\int_{-\infty}^{\infty} z^{4} e^{-U(z)} d z-\frac{h_{1}}{h_{0}} .
$$

Equations (5.33) are well-known; these are the Volterra-hierarchy investigated by M. Kac and P. von Moerbeke in 1975 [17] where they discovered the connection with the orthogonal polynomials as well. The partition function (5.1) appears to be the $\tau$-function of the Volterra-hierarchy (5.33) calculated on the special selfsimilar solution described by (5.34), (5.37). To illustrate this statement consider the case of the potential $U(z)=4 t z^{2}+4 z^{4}$. From (5.2) and (5.30) we have that

$$
\begin{aligned}
\frac{d}{d t} \ln \frac{Z_{n}}{Z_{n-1}} & =-\left(w_{n+1}+w_{n}\right), \\
\frac{d^{2}}{d t^{2}} \ln Z_{n} & =w_{n+1}\left(w_{n}+w_{n+2}\right)-w_{1} w_{2} .
\end{aligned}
$$

The comparison of these equalities with (3.24) and (3.25) and the arbitrariness in the definition of the $\tau$-function allow us to identify $Z_{n}$ and $\tau_{n}$. More precisely,

$$
Z_{n}(t)=\tau_{n}(t) \exp \left\{\int^{t} w_{1}(t) d t\right\}
$$

In addition to the discrete equations (1.8)-(1.10), the continuous limits (4.1) and (4.5) to the PI-equation, also arose in the work of $[10,11]$ where it was stated that the nonperturbative $2 \mathrm{D}$ gravity coupled to minimal conformal strings models, should be identified with the following double scaling limit of the matrix model (5.1):

$$
\begin{gathered}
\left(t_{1}, \ldots, t_{k}, \ldots\right) \doteqdot\left(\frac{\beta}{2}, \beta q_{1}, \ldots, \beta q_{k} \ldots\right) ; \beta, q_{k} \in \mathbb{R}, \\
\beta=h^{-4-2 / m} C_{1} \quad h \rightarrow 0 ; \quad \frac{n}{\beta}=\left(1+\delta \xi h^{4}\right) C_{2} .
\end{gathered}
$$

In the above, the integer " $m$ " numbers the models, " $\zeta$ " is a new independent variable and $C_{1}, \delta, C_{2}$ are some suitable real constants depended on the concrete 
choice of the potential $U(z)$ (i.e. on the parameters $\left.q_{1}, q_{2}, \ldots\right)$. In the language of quantum gravity the variable $\xi$ and constant $C_{2}$ are the renormalized string coupling and the critical value of the cosmological constant respectively. The constant $C_{1}$ is fixed by the assumption $(5.40 \mathrm{~b})$. The constant $\delta$ is the scaling parameter governing the value of coefficients in the limiting equations (5.41).

The authors of [10 and 11] assumed that at the above limits,

$$
w_{n} \cong \rho\left(1-2 h^{4 / m} u(\xi)\right), \quad w_{n \pm 1} \cong \rho\left(1-2 h^{4 / m} u\left(\xi \pm h^{2 / m}\right)\right),
$$

and then they showed that the discrete equation (5.6) (or (5.34)) for the function $w_{n}$, transforms into the following ordinary differential equation

$$
\left[H, A_{m}\right]=1, \quad m=2, \ldots
$$

for the function $u(\xi)$; here $H=-\frac{d^{2}}{d \xi^{2}}+u(\xi)$, and $A_{m}$ is the $A$-operator of the $m^{\text {th }}$ $\mathrm{KdV}$ equation. For $m=2$, Eq. (5.41) coincides with the PI-equation (1.4), and the double scaling limit (5.39), (5.40) is just the limit (4.1), (4.5).

The integer $m$ in (5.39)-(5.41) depends on the potential $U(z)$. All potentials which correspond to the same " $m$ " belong to the so called " $m^{\text {th }}$ universal class." The minimal potential in the $m^{\text {th }}$ universal class is an polynomial of order $2 m$. It is important that the limiting equation (5.41) depends only on the universal class of the potential $U(z)$.

The principal question, connected with the double scaling limit (5.39), (5.40), is how to characterize the solution $u(\xi)$ of the limiting equation (5.41). The answer to this question for the case $m=2$ and for the corresponding minimal potential $U(z)=t_{1} z^{2}+t_{2} z^{4}$ follows from the results of Sects. 3 and 4. Actually in this case the limit (5.39) is a reformulation of the following more fundamental limit [14]

$$
\left(t_{1}, t_{2}\right) \doteqdot\left(\frac{1}{2}, \frac{\lambda}{4 n}\right), \quad \lambda=\lambda_{c} e^{-\varepsilon \lambda_{1}^{0}}, \quad n=\varepsilon^{-5 / 4} e^{-\lambda_{2}^{0}}, \quad \varepsilon \rightarrow 0,
$$

where $\lambda_{c}=-\frac{1}{12}$ is the critical value of the cosmological constant and $\lambda_{1}^{0}, \lambda_{2}^{0}$ are the renormalized cosmological constant and renormalized string coupling respectively. The potential considered in Sects. 3 and 4 , is given by $U(z)=4 t z^{2}+4 z^{4}$ (recall $\left.t_{1}=4 t, t_{2}=4\right)$, while the physical case is given by $U(\hat{z})=\frac{\hat{z}^{2}}{2}+\frac{\lambda \hat{z}^{4}}{4 n}$. The change of variables $\hat{z}^{2}=4\left(\frac{n}{\lambda}\right)^{1 / 2} z^{2}$ implies that the two potentials are equivalent, and

$$
t=\frac{1}{2}\left(\frac{n}{\lambda}\right)^{1 / 2}
$$

Now we shall show that the physical limit (5.42) implies precisely the mathematical limit,

$$
t^{2}=-\frac{9}{2} h^{-5}, \quad \frac{n}{t^{2}}=-\frac{1}{3}\left(1+\frac{2}{3} h^{4} \xi\right)
$$

considered in Sect. 4. Indeed, $t^{2}=\frac{1}{4} \frac{n}{\lambda} \rightarrow \frac{e^{-\lambda_{2}^{0}}}{4 \lambda_{c}}\left(\varepsilon^{1 / 4}\right)^{-5}$, while $\frac{n}{t^{2}}=4 \lambda \rightarrow 4 \lambda_{c}\left(1-\varepsilon \lambda_{1}^{0}\right)$. 
Thus Eqs. (5.42) imply Eqs. (5.44), where

$$
h=\left(\frac{3}{2} e^{\lambda_{2}^{0}}\right)^{1 / 5} \varepsilon^{1 / 4}, \quad \xi=-\left(\frac{3}{2}\right)^{1 / 5} \lambda_{1}^{0} e^{-(4 / 5) \lambda_{2}^{0}}
$$

It is important to notice that the interesting physical limit involves considering integrals of the type $\int_{-\infty}^{\infty} e^{-\left(\hat{z}^{2} / z\right)-(\lambda / 4 n) \hat{z}^{4}} d z$, where $\frac{\lambda}{n}<0$. Our suggestion is to analytically continue these integrals and to consider instead,

$$
\int_{-\infty}^{\infty} \rightarrow s_{1} \int_{-\infty e^{i \pi / 4}}^{\infty e^{i \pi / 4}}-s_{3} \int_{-\infty e^{-i \pi / 4}}^{\infty e^{-i \pi / 4}} .
$$

It is clear that there exists a one parameter family of analytic continuations depending on the "regularization" parameters

$$
p \equiv-\frac{s_{3}}{s_{1}}
$$

Following the results of Sect. 4.2, we find, at the continuous limit, a one parameter family of the PI function $u(\xi)$. This family can be uniquely characterized by its asymptotics on the ray $\arg \xi=\pi-\frac{2 \pi}{5}$ :

$$
\begin{aligned}
& u(\xi)=e^{-i \pi / 5} \sqrt{\frac{|\xi|}{6}}+\gamma_{0}|\xi|^{-1 / 8} e^{(-8 i / 5)(3 / 2)^{1 / 4}|\xi|^{5 / 4}}+o\left(|\xi|^{-1 / 8}\right), \quad|\xi| \rightarrow \infty, \\
& \arg \xi=\pi-\frac{2 \pi}{5},
\end{aligned}
$$

where

$$
\gamma_{0}=\frac{-i}{\sqrt{8 \pi}} e^{\pi i / 20}\left(\frac{2}{3}\right)^{1 / 8} \frac{p}{1+p}, \quad p \neq-1
$$

Also, for real $\xi$

$$
u(\xi)=\sqrt{\frac{-\xi}{6}}+\cdots+\gamma(-\xi)^{-1 / 8} e^{(-8 / 5)(3 / 2)^{1 / 4}(-\xi)^{5 / 4}}(1+o(1)), \quad \xi \rightarrow-\infty
$$

where

$$
\gamma=\frac{i}{2} \frac{1}{\sqrt{8 \pi}}\left(\frac{2}{3}\right)^{1 / 8} \frac{1-p}{1+p}, \quad p \neq-1
$$

Also, using the formulae (A.8), (A.10) where one should put

$$
g_{1}=\frac{i}{1+p}, \quad g_{4}=\frac{i p}{1+p}
$$

it is possible to describe the behavior of $u(\xi)$ on all others "nonlinear" Stokes rays, that is $\arg \xi=\pi+\frac{2 \pi}{5}, \pi \pm \frac{4 \pi}{5}$.

Another example of a potential from the second $(m=2)$ universal class is given by the potential

$$
U(z)=t_{1} z^{2}+t_{2} z^{4}+t_{3} z^{6}
$$


In this case one finds [23] that the values of constants $C_{1}, C_{2}, \delta, \rho$ are:

$$
\begin{aligned}
\rho & =\frac{4\left(-q_{1}-\Delta\right)}{15 q_{2}}, \quad C_{2}=\frac{\rho^{2}}{4}\left(-q_{1}+2 \Delta\right), \quad \delta^{-1}=\frac{\left(-q_{1}+2 \Delta\right)}{2 \Delta}, \\
C_{1}^{-1} & =\rho^{2} \Delta, \quad \Delta=\frac{1}{2}\left(4 q_{1}^{2}-5 q_{2}\right)^{1 / 2}>0,
\end{aligned}
$$

where $q_{1}, q_{2}$ satisfy the inequalities

$$
q_{1}<0, \quad 0 \leqq 5 q_{2}<4 q_{1}^{2}
$$

Thus in this.case the relevant integrals converge and there appears no need for analytic continuation of the initial data (5.38). Nevertheless, following the methodology developed in Sects. 3, 4 one finds that the asymptotic representation (5.40) is consistent with the initial data (5.38) only if Eq. (5.38) is modified as in Eq. (5.46):

$$
\int_{-\infty}^{\infty} \rightarrow s_{1} \int_{-\infty}^{\infty e^{i \pi / 3}}-s_{3} \int_{-\infty e^{-i \pi / 3}}^{\infty e^{-i \pi / 3}} .
$$

After this modification the corresponding PI-function $u(\xi)$ is described by the same formulae (5.48)-(5.51).

Remarks.

(a) In order to have real solution $u(\xi)$ (and real initial data (5.38)) one needs $|p|=1$.

(b) The dots in (5.50) mean a perturbation series of the form $\sum_{l=1}^{\infty} c_{l}(-x)^{1 / 2-5 l / 2}$ (see [12]). The parameter $\gamma$ describes the nonperturbative effect. This effect is absent (as it follows from (5.51)) only for the case $p=1$.

(c) The fact that it is impossible to obtain the asymptotics (5.40) (for $m=2$ ) without the modification (5.54) confirms results of [24]. The "triply truncated" solution of PI considered in [24] corresponds in our notation to the case $p=0$.

In the case $5 q_{2}=4 q_{1}^{2}$ it was shown in [25] that the potential (5.52) belongs to the third class of universality, e.g. $m=3$. In this case instead of (1.4) one finds the equation

$$
-u_{\xi \xi \xi \xi}+20 u u_{\xi \xi}+10\left(u_{\xi}\right)^{2}-40 u^{3}=5 \xi
$$

and the following asymptotic behavior for $u(\xi)$ :

$$
u(\xi) \cong-\frac{1}{2} \xi^{1 / 3}, \quad \xi \rightarrow \pm \infty .
$$

It was claimed in [25] that this asymptotic defines the solution $u(\xi)$ uniquely. This was proved in [15] by the isomonodromic deformation technique. Using the results of [15] and the method described above one can verify that in the case $5 q_{2}=4 q^{2}$ the solution of (5.6) with initial data (5.38) (without any modification!) actually has asymptotics $(5.40)$ with $u(\xi)$ described by (5.55), (5.56).

We note that since $\gamma$ does not depend on the parameters $q_{1}, q_{2}$ the "universality" of the solution for the class of potentials (5.52) also takes place. It should also be mentioned that our calculations indicate that for all even $m$ a modification of the type (5.54) must be performed if one wants to obtain for the solution of (5.34) the asymptotic behavior (5.40). At the same time it seems that the theories with odd $m$ do not require this modification. We expect that our scheme for calculating the nonperturbative effects (parameter $\gamma$ ) can also be applied to the odd case. To 
achieve this one needs the description of the solutions of (5.55) in terms of the corresponding monodromy data (in analogy with the case of Eq. (1.4) studied in [22]). This description has been obtained in [28]. In this paper the nonperturbative term in the asymptotics (5.56) was also calculated:

$$
\begin{aligned}
u(\xi)= & -\frac{1}{2} \xi^{1 / 3}+\cdots, \quad \xi \rightarrow-\infty \\
u(\xi)= & -\frac{1}{2} \xi^{1 / 3}+\cdots+\frac{1}{\sqrt{\pi}}\left(\frac{5}{6}\right)^{1 / 8} \xi^{-1 / 4} \\
& \cdot \exp \left(-\sqrt[4]{30} \sin \left(\frac{1}{2} \operatorname{arct} g \frac{1}{\sqrt{5}}\right) \frac{6}{7} \xi^{7 / 6}\right) \\
& \cdot \cos \left(\sqrt[4]{30} \cos \left(\frac{1}{2} \operatorname{arct} g \frac{1}{\sqrt{5}}\right) \frac{6}{7} \xi^{7 / 6}-\frac{1}{4} \operatorname{arct} g \frac{1}{\sqrt{5}}\right)(1+o(1)) \quad \xi \rightarrow+\infty .
\end{aligned}
$$

\section{Appendix A}

According to the isomonodromy method the main role in the investigation of the PI-equation (1.4) is played by the second equation in (4.12). This equation has two singular points; a regular singularity at $\eta=0$ and an irregular at $\eta=\infty$. Following [22] we shall introduce the monodromy data for the second equation in (4.12) as the set of Stokes matrices $G_{j}, j \in \mathbb{Z}$, defined by the equations

$$
\mathbb{G}_{j}=\left[Y^{j}(\kappa)\right]^{-1} Y^{j+1}(\kappa) .
$$

Here $Y^{j}(\kappa), j \in \mathbb{Z}$ are the canonical solutions determined by the asymptotics

$$
\begin{aligned}
& Y^{j}(\kappa)=\left(I+O\left(\frac{1}{\kappa}\right)\right) \exp \left\{\sigma_{3}\left(\frac{4}{5} \kappa^{5}+\xi \kappa\right)\right\}, \\
& k \rightarrow \infty \text { in } \frac{\pi}{5}\left(j-\frac{1}{2}\right) \leqq \arg \kappa<\frac{\pi}{5}\left(j+\frac{1}{2}\right) .
\end{aligned}
$$

The Stokes matrices $\mathbb{G}_{j}$ have the usual triangular structure

$$
\mathbb{G}_{2 l+1}=\left(\begin{array}{cc}
1 & g_{2 l+1} \\
0 & 1
\end{array}\right), \quad \mathbb{G}_{2 l}=\left(\begin{array}{cc}
1 & 0 \\
g_{2 l} & 1
\end{array}\right)
$$

and they satisfy the relations

$$
\mathbb{G}_{j+5}=\sigma_{1} \mathbb{G}_{j} \sigma_{1}, \quad j \in \mathbb{Z} ; \quad \mathbb{G}_{1} \mathbb{G}_{2} \mathbb{G}_{3} \mathbb{G}_{4} \mathbb{G}_{5}=i \sigma_{1} .
$$

This implies that the monodromy data for the second equation in (4.12) can be parametrized by the Stokes multipliers $\left\{g_{j}\right\}_{j=1}^{5}$ connected by the relations

$$
g_{5}=i\left(1+g_{2} g_{3}\right), g_{3}+g_{1}\left(1+g_{2} g_{3}\right)=i, \quad g_{4}=i\left(1+g_{1} g_{2}\right) \text {. }
$$

The monodromy data $\left\{g_{j}\right\}_{1}^{5}$ provide a parametrization of the solutions of the PI-equation (1.4). An alternative parametrization is provided by the asymptotic characteristics of the solution $u(\xi)$ on one of the "nonlinear Stokes rays," given by

$$
\arg \xi=\pi, \quad \pi \pm \frac{2 \pi}{5}, \quad \pi \pm \frac{4 \pi}{5}
$$


The main result of [22] is the calculation of the explicit form of the connection between these two parametrization. In particular, for the special case

$$
g_{5}=0
$$

and, as a consequence,

$$
g_{3}=i, \quad g_{2}=i, \quad g_{4}+g_{1}=i
$$

the following asymptotic behavior for $u(\xi)$ has been obtained:

$$
\begin{aligned}
\arg \xi= & \pi-\frac{2 \pi}{5}: u(\xi)=e^{-i \pi / 5} \sqrt{\frac{|\xi|}{6}}-\frac{e^{i \pi / 20}}{\sqrt{8 \pi}}\left(\frac{2}{3}\right)^{1 / 8} g_{4}|\xi|^{-1 / 8} \\
& \cdot \exp \left\{\frac{i 8}{5}\left(\frac{3}{2}\right)^{1 / 4}|\xi|^{5 / 4}\right\}+o\left(|\xi|^{-1 / 8}\right), \\
\arg \xi= & \pi+\frac{2 \pi}{5}: u(\xi)=e^{i \pi / 5} \sqrt{\frac{|\xi|}{6}}+\frac{e^{-i \pi / 20}}{\sqrt{8 \pi}}\left(\frac{2}{3}\right)^{1 / 8} g_{1}|\xi|^{-1 / 8} \\
& \cdot \exp \left\{\frac{-8 i}{5}\left(\frac{3}{2}\right)^{1 / 4}|\xi|^{5 / 4}\right\}+o\left(|\xi|^{-1 / 8}\right) .
\end{aligned}
$$

Moreover, in [22] using ideas based on the analytical continuation of the asymptotics (A.7), (A.8), the following description of the asymptotics of the solution $u(\xi)$ on the ray $\arg \xi=\pi$ is proposed:

$$
u(\xi)=\sqrt{\frac{-\xi}{6}}+\cdots+\frac{1}{\sqrt{8 \pi}}\left(\frac{2}{3}\right)^{1 / 8} \frac{g_{1}-g_{4}}{2}|\xi|^{-1 / 8} \exp \left\{-\frac{8}{5}\left(\frac{3}{2}\right)^{1 / 4}(-\xi)^{5 / 4}\right\}(1+o(1)) \text {. }
$$

The behavior of the function $u(\xi)$ on the rays $\arg \xi=\pi \pm \frac{4 \pi}{5}$ is more complicated. It depends on the combinations

$$
1+i g_{1}\left(\operatorname{ray} \arg \xi=\pi-\frac{4 \pi}{5}\right) \text { and } 1+i g_{4}\left(\operatorname{ray} \arg \xi=\pi+\frac{4 \pi}{5}\right) .
$$

For example, if $1+i g_{1}=0$ (i.e. $g_{1}=i, g_{4}=0$ ), the asymptotics of $u(\xi)$ on the rays $\arg \xi=\pi \mp \frac{4 \pi}{5}$ are the following:

$$
\begin{aligned}
\arg \xi= & \pi-\frac{4 \pi}{5}: \\
u(\xi)= & e^{-2 i \pi / 5} \sqrt{\frac{|\xi|}{6}}+\cdots+\frac{e^{-9 i \pi / 10}}{\sqrt{8 \pi}}\left(\frac{2}{3}\right)^{1 / 8} \frac{1}{2}|\xi|^{-1 / 8} \\
& \cdot \exp \left\{-\frac{8}{5}\left(\frac{3}{2}\right)^{1 / 4}|\xi|^{5 / 4}\right\}(1+o(1))
\end{aligned}
$$




$$
\begin{aligned}
\arg \xi & =\pi+\frac{4 \pi}{5}: \\
u(\xi) & =-e^{2 i \pi / 5} \sqrt{\frac{|\xi|}{6}}+\frac{e^{-7 i \pi / 20}}{\sqrt{8 \pi}}\left(\frac{2}{3}\right)^{1 / 8}|\xi|^{-1 / 8} \exp \left\{\frac{i 8}{5}\left(\frac{3}{2}\right)^{1 / 4}|\xi|^{5 / 4}\right\}+o\left(|\xi|^{-1 / 8}\right) .
\end{aligned}
$$

For details and explicit formulae for the cases $1+i g_{1}>0$ and $1+i g_{4} \geqq 0$ we refer to [22].

As it follows from the asymptotic formulae (A.7-A.11), in the case $g_{5}=0=g_{4}$, $g_{1}=g_{2}=g_{3}=i$ we obtain the so-called "triply truncated solution," the solution having infinitely many poles only in the sector $\frac{7}{5} \pi<\arg \xi<\frac{9}{5} \pi$.

\section{Appendix B}

Here we prove that the Lax-type equation (5.34) coincides with equations (5.26). Similarly it can be shown that Eq. (5.33) coincides with Eq. (5.25).

The equalities

imply

$$
\left(U_{-}^{\prime}(L)\right)_{n n}=\left(U^{\prime}(L)\right)_{n n}=0
$$

$$
\left[L, U_{-}^{\prime}(L)\right]_{n, m}=\left[L, U^{\prime}(L)\right]_{n, m}=0, \quad \forall n \neq m .
$$

Also for the diagonal elements we have

$$
\left(L U_{-}^{\prime}(L)\right)_{n, n}=L_{n, n+1}\left(U_{-}^{\prime}(L)\right)_{n+1, n}=\left(U_{-}^{\prime}(L)\right)_{n+1, n} L_{n, n+1}=\left(U_{-}^{\prime}(L) L\right)_{n+1, n+1},
$$

so Eq. (5.34) can be rewritten as

$$
I_{n+1}-I_{n}=1
$$

where we introduce the notation

$$
I_{n} \doteqdot\left(U_{-}^{\prime}(L) L\right)_{n, n} .
$$

The right-hand side of (5.26) can be transformed as follows:

$$
\begin{aligned}
\sum_{k=1}^{2 n} 2 k t_{k} L_{n n}^{2 k} & =\left(U^{\prime}(L) L\right)_{n n}=U^{\prime}(L)_{n, n-1} L_{n-1, n}+U^{\prime}(L)_{n, n+1} L_{n+1, n} \\
& =U^{\prime}(L)_{n, n-1} L_{n-1, n}+L_{n, n+1} U^{\prime}(L)_{n+1, n}=\left(U_{-}^{\prime}(L) L\right)_{n, n}+\left(L U_{-}^{\prime}(L)\right)_{n, n},
\end{aligned}
$$

hence Eq. (5.26) can be rewritten as

Note that

$$
I_{n+1}+I_{n}=1+2 n \text {. }
$$

$$
I_{n}=\left(U_{-}^{\prime}(L)\right)_{n, n-1} L_{n-1, n}=\frac{1}{2} w_{n}^{1 / 2}\left(U_{-}^{\prime}(L)\right)_{n, n-1}=\frac{1}{2} w_{n}^{1 / 2}\left(U^{\prime}(L)\right)_{n, n-1} .
$$

This equation shows that $I_{0}=0$ implies $w_{0}=0$. Also, $I_{0}=0$ together with the condition $w_{0}=0$ for $n<0$ implies that both (B.1) and (B.2) coincide with $I_{n}=n, n \geqq 0$.

Acknowledgements. We would like to thank A. Migdal and G. Moore for valuable discussions, as well as S. P. Novikov, V. E. Korepin and L. A. Takhtajan for their encouragement. This work was partially supported by the National Science Foundation under Grant Number DMS-8803471, and Air Force Office of Scientific Research under Grant Number 87-0310. 


\section{References}

1. Painlevé, P.: Bull. Soc. Math. Fr. 28, 214 (1900); Acta Math. 25, 1 (1902); Gambier, B.: Acta Math. 33, 1 (1909)

2. Ince, E. L.: Ordinary Differential Equations, (1927). New York: Dover 1956

3. Barouch, E., McCoy, B. M., Wu, T. T.: Phys. Rev. Lett. 31, 1409 (1973); Wu, T. T., McCoy, B. M., Tracy, C. A., Barouch, E.: Phys. Rev. B13, 316 (1976)

4. Jimbo, M., Miwa, T., Mori, Y., Sato, M.: Physica D1, 80 (1980); Jimbo, M., Miwa, T.: Proc. Jpn. Acad. A56, 405 (1980)

5. Ablowitz, M. J., Segur, H.: Phys. Rev. Lett. 38, 1103 (1977)

6. Flaschka, H., Newell, A. C.: Commun. Math. Phys. 76, 67 (1980)

7. Ueno, K.: Proc. Jpn. Acad. A56, 97 (1980); Jimbo, M., Miwa, T., Ueno, K.: Physica D2, 306 (1981); Jimbo, M., Miwa, T.: Physica D2, 407 (1981); 4D, 47 (1981); Jimbo, M.: Prog. Theor. Phys. 61, 359 (1979)

8. Fokas, A. S., Xin Zhou,: On the Solvability of Painleve II and IV, Clarkson University, preprint INS \#148, January 1990

9. Brézin, E., Kazakov, V.: Phys. Lett. B236, 144 (1990)

10. Douglas, M., Shenker, S.: Strings in Less than One Dimension, Rutgers preprint RU-89-34

11. Gross, D., Migdal, A.: Phys. Rev. Lett. 64, 127 (1990)

12. Gross, D., Migdal, A.: A Nonperturbative Treatment of Two-Dimensional Quantum Gravity, Princeton preprint PUPT-1159 (1989)

13. Douglas, M.: Strings in Less than One Dimension and the Generalized KdV Hierachies, Rutgers preprint RU-89-51

14. Witten, E.: Two Dimensional Gravity and Intersection Theory on Moduli Space, IAS preprint, IASSNS-HEP-90/45

15. Moore, G.: Geometry of the String Equations, Yale preprint YCTP-P4-90

16. Moore, G.: Matrix Models of 2D Gravity and Isomonodromic Deformation, YCTP-P17-90, RU-90-53

17. Kac, M., von Moerbeke, P.: Adv. Math. 16, 160-164 (1975)

18. Fokas, A. S., Manakov, S. V.: Phys. Lett. A150, 369 (1990)

19. Nijhoff, F. W., Papageorgiou, V. G.: Similarity Reductions of Integrable Lattices and Discrete Analogues of Painlevé II Equation, INS \#155, October 1990

20. McCoy, M., Perk, H. H., Shrock, R. E.: Correlation Functions of the Transverse Ising Chain at the Critical Field for Large Temporal and Spatial Variations, ITP S13-83-5 (1983)

21. Fokas, A. S., Mugan, U., Ablowitz, M. J.: Physica D30, 247 (1988)

22. Kapaev, A. A.: Asymptotics of Solutions of the Painleve Equation of the First Kind, Differential Equations, 24 (1988) (Russian)

23. Its, A. R., Kitaev, A. V.: Mathematical Aspects of 2D Quantum Gravity, MPLA, 5 (25), 2079 (1990)

24. David, F.: Loop Equations and Non-Perturbative Effects in Two-Dimensional Quantum Gravity. MPLA, 5 (13), 1019-1029 (1990)

25. Brézin, E., Marinari, E., Parisi, G.: A Non-Perturbative Ambiguity Free Solution of a String Model, Roma preprint ROM2F-90-09

26. Bessis, D., Itzykson, C., Zuber, I. B.: Quantum Field Theory Techniques in Graphical Enumeration. Adv. Appl. Math. 1, 109-157 (1980)

27. Its, A., Novokshenov, V. Yu.: The Isomonodromic Deformation Method in the Theory of Painlevé Equations. Lecture Notes Mathematics, vol. 1191. Berlin, Heidelberg, New York: Springer 1986

28. Kapaev, A. A.: Quasinonlinear Solution of the Equations $P_{1}^{2}$, Zap. Sem. LOMI 187, 88-110 (1990)

29. Fokas, A. S., Its, A. R., Kitaev, A. V.: The Isomonodromic Approach in the Theory of Two Dimensional Quantum Gravity. Uspehi Matem. Nauk. 45 (6), 135-136 (1990) (Russian) 